\title{
Unified description of fission in fusion and spallation reactions
}

\author{
Davide Mancusi, ${ }^{1, *}$ Robert J. Charity, ${ }^{2}$ and Joseph Cugnon ${ }^{2}$ \\ ${ }^{1}$ AGO Department, University of Liège, Allée du 6 Août 17, Bât. B5, B-4000 Liège 1, Belgium \\ ${ }^{2}$ Department of Chemistry, Washington University, St. Louis, Missouri 63130, USA
}

(Received 16 July 2010; published 21 October 2010)

\begin{abstract}
We present a statistical-model description of fission, in the framework of compound-nucleus decay, which is found to simultaneously reproduce data from both heavy-ion-induced fusion reactions and proton-induced spallation reactions at around $1 \mathrm{GeV}$. For the spallation reactions, the initial compound-nucleus population is predicted by the Liège intranuclear cascade model. We are able to reproduce experimental fission probabilities and fission-fragment mass distributions in both reactions types with the same parameter sets. However, no unique parameter set was obtained for the fission probability. The introduction of fission transients can be offset by an increase of the ratio of level-density parameters for the saddle-point and ground-state configurations. Changes to the finite-range fission barriers could be offset by a scaling of the Bohr-Wheeler decay width as predicted by Kramers. The parameter sets presented allow accurate prediction of fission probabilities for excitation energies up to $300 \mathrm{MeV}$ and spins up to $60 \hbar$.
\end{abstract}

DOI: 10.1103/PhysRevC.82.044610

PACS number(s): 21.10.Ma, 24.60.Dr, 25.40.Sc, 25.70.Jj

\section{INTRODUCTION}

Although 70 years have passed since the seminal works of Bohr and Wheeler [1] and Weisskopf and Ewing [2] and the establishment of a qualitative understanding of the de-excitation mechanism of excited nuclei, quantitatively accurate and universally applicable models do not yet exist. This is partly because of the vast number of nuclear data that must be fed into the models and partly because of the uncertainties in the fundamental ingredients of de-excitation, such as level densities and emission barriers. Even the choice of the mathematical formalism, however, is not devoid of confusion, as already pointed out by Moretto [3] and Swiatecki [4].

One way to lift the degeneracy of the ingredients of the model is to explore diverse regions of the compound-nucleus parameter space. A systematic study of nuclei with different masses, excitation energies, spins, and isospins would be sensitive to most of the assumptions of the de-excitation model. The long-term goal of such an investigation would be to identify a minimal set of physical ingredients necessary for a unified quantitative description of nuclear de-excitation chains.

The production of excited compound nuclei can proceed from several entrance reactions. There has been a long history of compound-nucleus studies using heavy-ion-induced fusion reactions. These reactions allow one to specify the compound-nucleus mass, charge, and excitation energy; however, a distribution of compound-nucleus spins is obtained. Statistical-model parameters such as fission barriers are quite sensitive to spin. Heavy-ion-induced fission probabilities, evaporation spectra, and residue masses can generally be reproduced in statistical-model calculations. However, some fine-tuning of the statistical-model parameters to the mass region or reaction is often needed. A unified description over

\footnotetext{
*d.mancusi@ulg.ac.be
}

all mass regions is still lacking even for these reactions. Some work toward this, concentrating on the parameters describing the shape of the evaporation spectra, is presented in Ref. [5].

Another typical entrance channel for the production of excited compound nuclei is spallation. The present interest in spallation derives mainly from the applications to acceleratordriven systems, namely, accelerator-based reactors for the transmutation of nuclear waste. At incident energies relevant for transmutation (a few hundred mega-electron volts), an appropriate theoretical tool for the description of protonnucleus reactions is the coupling of an intranuclear-cascade model with a nuclear de-excitation model. It is assumed in the intranuclear-cascade-model framework that the incoming particle starts an avalanche of binary collisions with and between the target nucleons. When the cascade stage ends, an excited and thermalized remnant is formed, with a basically unchanged density. In the subsequent de-excitation stage, the remnant gets rid of the excess energy by particle evaporation and/or fission. For these reactions, the need for a model to predict the initial compound-nucleus mass, charge, excitation, and spin distributions adds some uncertainty in our ability to constrain the statistical-model parameters by fitting data. However, spallation reactions allow us to explore different regions of compound-nucleus spin and excitation energy than can be probed with fusion reactions alone and thus can be important in parameter fitting.

The role of a transient fission width is currently somewhat controversial. Fission transients are where the fission decay width is not constant, but increases from 0 toward its equilibrium value [6]. Fission transients were first introduced to help explain the large number of neutrons emitted from a fissioning system before the scission point was reached [7]. The statistical model assumes that there is an equilibrium in all degrees of freedom including the deformation degrees of freedom associated with fission. If all compound nuclei have spherical shapes initially, then they cannot instantaneously fission, as it takes a finite time to diffuse toward the saddle and, 
subsequently, the scission point. The transient time, the time scale necessary for the system to explore large fluctuations in the deformation degrees of freedom, is a function of the viscosity of the nucleus. The predicted fission probability is also very sensitive to the assumed initial deformation [8], which may depend on the entrance channel.

The transient time is often called a fission delay, as fission is suppressed during this period. If the excitation energy of the compound nucleus is high enough, then there will be a probability of emitting a light or, possibly, even an intermediate-mass fragment during the fission delay. Neutron emission lowers the excitation energy, and charged-particle emission also lowers the fissility of the nucleus by increasing its fission barrier. These effects will lead to a reduced fission probability after the fission delay is over. An experimental test of this idea would be the observation of reduced fission probability or enhanced evaporation-residue survival at high excitation energies that cannot be explained in terms of the statistical model. At present there is some controversy over the need for fission transients.

A number of theoretical studies [9] reproduce experimental fission probabilities and pre-scission neutron multiplicities with transient fission widths when the viscosity increases with the mass of the compound nucleus. Transient fission has also been invoked to explain the relatively large number of evaporation residues measured for the very fissile ${ }^{216} \mathrm{Th}$ compound nuclei formed in ${ }^{32} \mathrm{~S}+{ }^{184} \mathrm{~W}$ reactions, compared to a statistical-model prediction [10]. Alternatively other studies have reproduced fission probabilities with no transient effects [11], and Lestone and McCalla [12] suggest that fission transients are unimportant whenever the nuclear temperature is less than the fission barrier. Similarly, with data of a very high excitation energy obtained in $2.5-\mathrm{GeV}$ proton-induced reactions, no transients were needed to reproduce the measured fission yields [13].

This paper discusses the application of the GEMINI++ de-excitation model [14] to the description of fission in fusion and spallation reactions. In the latter case, the description of the entrance channel is provided by a coupling to the Liège intranuclear cascade (INCL) model [15]. Both INCL and GEMINI++ are among the most sophisticated models in their respective fields. The present work also represents the first thorough discussion of their coupling.

We compare the predictions of the models with experimental residue yields in spallation studies and with fission and evaporation-residue excitation functions measured in heavyion-induced fusion reactions. The choice of the observables was motivated by considerations about their sensitivity to fission and by the availability of experimental data. An exhaustive discussion should, of course, take into account other observables (e.g., double-differential particle spectra) and the competition of fission with other de-excitation channels, but this is outside the scope of the present paper. Therefore, we describe how the parameters of the statistical-decay model have been consistently adjusted to reproduce the data and discuss to what extent a successful unified description of these reactions has been achieved. Finally, we explore whether all the data can be described within the statistical model or whether transient fission decay widths are needed.

\section{THE MODELS}

We now turn to the description of the most important features of the models we have considered. The codes are not analyzed in detail; only the most important features are outlined.

\section{A. GEMINI++}

GEMINI++ is an improved version of the GEMINI statistical decay model, developed by R. J. Charity [16] with the goal of describing complex-fragment formation in heavy-ion fusion experiments. The de-excitation of the compound nucleus proceeds through a sequence of binary decays until particle emission becomes energetically forbidden or improbable owing to competition with $\gamma$-ray emission.

Because compound nuclei created in fusion reactions are typically characterized by large intrinsic angular momenta, the GEMINI and GEMINI++ models explicitly consider the influence of spin and orbital angular momentum on particle emission. Moreover, GEMINI and GEMINI++ do not restrict binary-decay modes to nucleon and light-nucleus evaporation, which are the dominant decay channels, but allow the decaying nucleus to emit a fragment of any mass. The introduction of a generic binary-decay mode is necessary for the description of complex-fragment formation and is one of the features that set GEMINI and GEMINI++ apart from most of the other de-excitation models.

Emission of nucleons and light nuclei $(Z \leqslant 2,3$, or 4 , depending on the user's choice) is described by the HauserFeshbach evaporation formalism [17], which explicitly treats and conserves angular momentum. The production of heavier fragments is described by Moretto's binary-decay formalism [3]. However for symmetric divisions of heavy compound nuclei, the Moretto formalism employing Sierk's finite-range calculations $[18,19]$ fails to reproduce the mass distribution of decay products (Sec. IIIC). However, for light systems, the Moretto formalism works quite well $[16,18,20]$ and is still used in GEMINI++. Also, for the heavier systems, but for mass asymmetries outside of the symmetric fission peak, the Moretto formalism is still used. Otherwise, the total fission yield is obtained from the Bohr-Wheeler formalism [1] and the width of the fission-fragment mass distribution is taken from systematics compiled by Rusanov et al. [21] (see Sec. III C).

Table I summarizes the de-excitation mechanisms featured by GEMINI++. The parameters of the model associated with evaporation have been adjusted to reproduce data from heavyion-induced fusion reactions. This is described in Ref. [5]

TABLE I. List of de-excitation processes featured by the GEMINI ++ model. $Z$ represents the charge number of the emitted particle and $Z_{\text {switch }}$ can be chosen to be 2,3 , or 4 .

\begin{tabular}{llc}
\hline \hline Process & \multicolumn{1}{c}{ Model } & \multicolumn{1}{c}{ Note } \\
\hline $\begin{array}{l}\text { Light-particle } \\
\text { evaporation } \\
\text { Binary decay }\end{array}$ & Hauser-Feshbach [17] & $Z \leqslant Z_{\text {switch }}$ \\
$\begin{array}{l}\text { Fission } \\
\text { Partition in fission }\end{array}$ & Bohr-Whetto [3] & $Z>Z_{\text {switch }}$ \\
\hline \hline
\end{tabular}


in more detail, but we briefly list the important adjustments for that work. To fit experimental light-particle kinetic-energy spectra, the transmission coefficients in the Hauser-Feshbach formalism were calculated for a distribution of Coulomb barriers associated with thermal fluctuations. The nature of fluctuations is not entirely clear; they may be fluctuations in compound-nucleus shape and/or its density and/or its surface diffuseness.

Level densities were calculated with the Fermi-gas form:

$$
\rho\left(E^{*}, J\right) \sim \exp (2 \sqrt{a(U) U}),
$$

where $E^{*}$ is the total excitation energy, $J$ is the spin, and $U$ is the thermal excitation energy after the pairing, rotational, and deformation energies are removed. The level-density parameter used should be considered an effective value, as no collective-enhancement factors are used in the level-density formula in Eq. (1).

The level-density parameter $a(U)$ is excitation energy dependent, with an initial fast dependence owing to the washing-out of shell effects following Ref. [22] and the slower dependence needed to fit the evaporation spectra. The shell-smoothed level-density parameter was assumed to have the form

$$
\widetilde{a}(U)=\frac{A}{k_{\infty}-\left(k_{\infty}-k_{0}\right) \exp \left(-\frac{\kappa}{k_{\infty}-k_{0}} \frac{U}{A}\right)},
$$

which varies from $A / k_{0}$ at low excitation energies to $A / k_{\infty}$ at high values. Here $k_{0}=7.3 \mathrm{MeV}$, consistent with neutronresonance counting data at excitation energies near the neutron separation energy, and $k_{\infty}=12 \mathrm{MeV}$. The parameter $\kappa$ defines the rate of change of $\widetilde{a}$ with energy and it is essentially zero for light nuclei (i.e., a constant $\widetilde{a}$ value) and increases roughly exponentially with $A$ for heavier nuclei. Although we expect a decrease in the level-density parameter with $U$, owing to decreasing importance of long-range correlations with increasing excitation energy (caused by washing-out of collective enhancement factors and, also, the reduction in the intrinsic level-density parameter), the strong mass dependence cannot be explained at present.

The strong excitation-energy dependence of $\widetilde{a}$ for heavy nuclei leads to increased nuclear temperatures, which enhance very weak decay channels. For very fissile systems, these weak decay channels include $n, p$, and $\alpha$ evaporation, and thus GEMINI++ calculations predict enhanced evaporation-residue production consistent with some experimental data. These enhanced evaporation-residue yields had previously been interpreted as a consequence of transient fission [10]. Clearly the excitation-energy dependence of $\widetilde{a}$ is very important in understanding the role of transient fission. However, we note that for fission, the dominant decay mode in fissile nuclei, the yield is decreased relatively little by the increased temperature.

\section{B. INCL4.5}

The INCL model [15] can be applied to collisions between nuclei and pions, nucleons or light nuclei of energy lower than a few giga-electron volts. The particle-nucleus collision is modelled as a sequence of binary collisions among the particles present in the system; particles that are unstable over the time scale of the collision, notably $\Delta$ resonances, are allowed to decay. The nucleus is represented by a square potential well whose radius depends on the nucleon momentum; thus, nucleons move in straight lines until they undergo a collision with another nucleon or until they reach the surface, where they escape if their total energy is positive and they manage to penetrate the Coulomb barrier.

The latest version of the INCL model (INCL4.5) includes, among other things, isospin- and energy-dependent nucleon potentials, an isospin-dependent pion potential, and a new dynamical coalescence algorithm for the production of light clusters (up to $A=8$ with the default program options). A comprehensive description will be published in the near-future [23].

The INCL model simulates a complete cascade event, its output being the velocities of all the emitted particles. The characteristics of the remnant (its mass, charge, momentum, excitation energy, and intrinsic angular momentum) are derived from the application of conservation laws and are passed to the chosen de-excitation code; the latter simulates the decay of the remnant into a nuclear-stable residue plus a number of nucleons, nuclei, and/or $\gamma$ rays.

The INCL4.5 model is not to be considered an adjustable model. It does contain parameters, but they either are taken from known phenomenology (such as the matter density radius of the nuclei) or have been adjusted once for all (such as the parameters of Pauli blocking or those that determine the coalescence module for the production of light charged clusters). Therefore adjusting INCL4.5/GEMINI ++ for the experimental data basically amounts to the adjustment of the GEMINI++ parameters. One should keep in mind that INCL4.5 brings in its own physics features and limitations. For our purpose here, they essentially determine the distributions of the remnant properties. These quantities cannot be compared directly with experimental data, but the predictions of INCL4.5 concerning those observables that can be compared directly to experiment, namely, the high-energy parts of particle spectra, are of rather good quality, as shown recently [24].

\section{ADJUSTMENT OF FISSION YIELDS}

The assumption of thermal equilibrium implied by the statistical-decay hypothesis implies that the excited nucleus cannot retain any memory of the entrance channel. One of the main aspirations of the GEMINI ++ development is to provide a unified and coherent description of nuclear de-excitation in spallation and fusion reactions at the same time.

The degrees of freedom in the model induce different characteristic dependencies of the fission width on the remnant spin and excitation energy, because fission is, at the very least, sensitive to spin, the fade-out of shell and collective effects, level densities, and fission barriers. Because variations in some of the free parameters can produce similar effects, it is difficult to disentangle the various contributions and to put stringent constraints on the de-excitation model just by looking at experiments of a single type. However, fusion and spallation reactions populate different regions of the compound-nucleus spin/excitation-energy plane. A comparison of the populations in the $E^{*}-J$ plane is shown in Fig. 1 for the $1-\mathrm{GeV} p+{ }^{208} \mathrm{~Pb}$ spallation reaction and the ${ }^{19} \mathrm{~F}+{ }^{181} \mathrm{Ta} \rightarrow{ }^{200} \mathrm{~Pb}$ fusion 


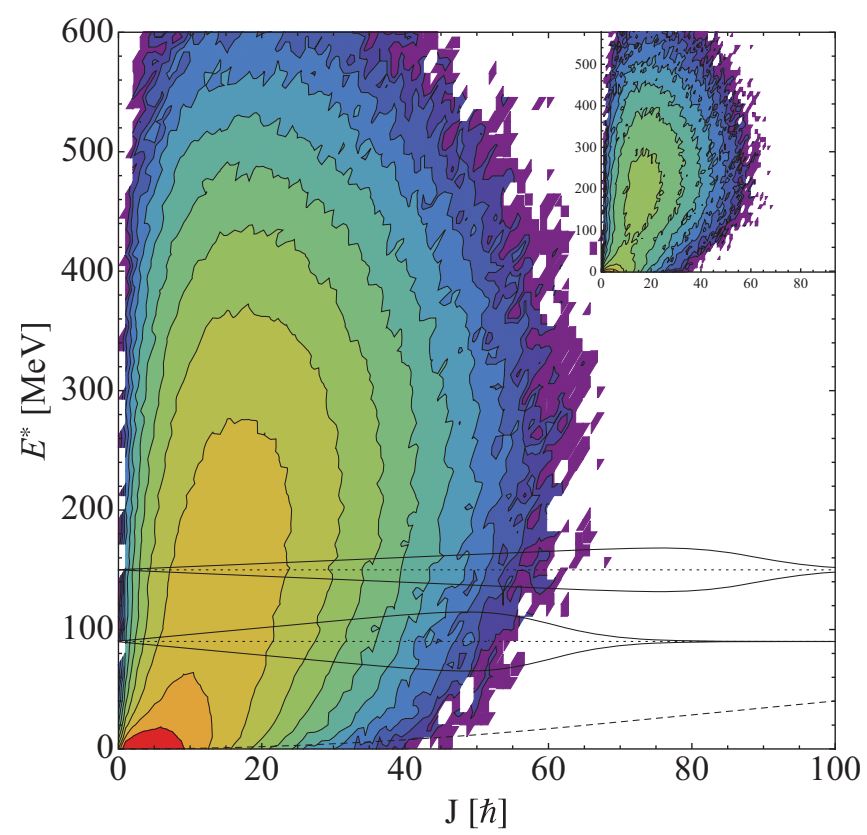

FIG. 1. (Color online) Comparison of the distributions of excitation energy and spin populated in the ${ }^{19} \mathrm{~F}+{ }^{181} \mathrm{Ta} \rightarrow{ }^{200} \mathrm{~Pb}$ fusion reaction for $E^{*}=90$ and $150 \mathrm{MeV}$ (horizontal lines) with the INCL4.5 prediction for the $1-\mathrm{GeV} p+{ }^{208} \mathrm{~Pb}$ spallation reaction (contours, logarithmically spaced). Inset: Distribution of fissioning remnants for the spallation reaction (same contour levels). The dashed line is the macroscopic yrast line from Sierk [25].

reaction. The spallation population is represented by the contours. Examples of fusion distributions for $E^{*}=90$ and $150 \mathrm{MeV}$ are represented by pairs of symmetrical solid lines; the distance between the solid lines is proportional to the population. The inset shows the distributions of remnants leading to fission in the case of spallation; the contour levels are the same as in the main plot. As a guide for ${ }^{200} \mathrm{~Pb}$, the macroscopic yrast line from Sierk [25] is indicated.

For the spallation reaction, the INCL 4.5 model predicts average values of about $167 \mathrm{MeV}$ and $16.5 \hbar$, but both distributions are quite broad and extend up to $\sim 650 \mathrm{MeV}$ and $\sim 50 \hbar$, respectively. In contrast, the fusion reactions we considered are characterized by higher spins and lower excitation energies. We concentrate only on complete-fusion reactions, where the excitation energy of the compound nucleus is defined entirely from energy conservation. The requirement of complete fusion restricts us to projectile bombarding energies of less then $10 \mathrm{MeV} / A$, where incomplete fusion and the pre-equilibrium process are small. We can explore somewhat higher excitation energies with more symmetric entrance channels, but high spins will still be populated. Thus it is clear that the comparison between spallation and fusion data represents a promising tool for extending the predictive power of the model over a wide region of mass, energy, and spin.

The outlined simultaneous-fitting strategy would be meaningless if the two types of data probed separate modelparameter subsets; separate fits would then be just as effective as a combined fit. This is not the case, as Fig. 1 suggests. Spallation is characterized by a large spread in the compound- nucleus excitation energy; however, the excitation-energy dependence of fission can be tested in fusion reactions too, using excitation curves. Thus, both types of data sets are probably sensitive to the model parameters connected with the excitation-energy dependence of fission. A similar argument can be produced for the model parameters connected with the spin dependence. The model parameters that are sensitive to the mass and charge of the fissioning nucleus are probed by spallation through the distribution of cascade remnants and by fusion, when one considers neighboring compound nuclei. We thus conclude that fusion and spallation probe different, but overlapping areas of the model-parameter space. Under these conditions, simultaneous fitting is most likely advantageous.

The fusion reactions used in this study are listed in Table II with the range of excitation energies probed and the appropriate references for the data. In most cases we have selected data where both the evaporation-residue and the fission cross sections have been determined. The sum of these two quantities gives the total fusion cross section and this is used to constrain the compound-nucleus spin distribution. We assume that the spin distribution has a roughly triangular shape characterized by a maximum value $J_{0}$ with a smooth cutoff characterized by the parameter $\delta J$, that is,

$$
\sigma_{\text {fus }}(J)=\pi \lambda^{2}(2 J+1) \frac{1}{1+\exp \left(\frac{J-J_{0}}{\delta J}\right)} .
$$

The parameter $J_{0}$ is determined from the total fusion cross section

$$
\sigma_{\text {fus }}=\sum_{J=0}^{\infty} \sigma_{\text {fus }}(J),
$$

and $\delta J$ is set to values from 3 to $10 \hbar$, with larger values associated with heavier projectiles. These values are similar to estimates obtained in Refs. [26-28].

Generally the fission cross section is only sensitive to the value of $\delta J$ at excitation energies where the fission probability is low and rises rapidly with $J$ [26]. The values of $\delta J$ assumed in the following calculations are also listed in Table II.

TABLE II. Experimental fission and evaporation-residue data used in this work.

\begin{tabular}{lccccc}
\hline \hline $\mathrm{CN}$ & Reaction & $\begin{array}{c}E^{*} \text { range } \\
(\mathrm{MeV})\end{array}$ & $\sigma_{\mathrm{ER}}$ & $\sigma_{\text {fus }}$ & $\begin{array}{c}\delta J \\
(\hbar)\end{array}$ \\
\hline${ }^{156} \mathrm{Er}$ & ${ }^{64} \mathrm{Ni}+{ }^{90} \mathrm{Zr}$ & $25-82$ & {$[29]$} & {$[30]$} & 10 \\
${ }^{158} \mathrm{Dy}$ & ${ }^{19} \mathrm{~F}+{ }^{139} \mathrm{La}$ & $68-94$ & {$[26]$} & {$[26]$} & 2.3 \\
${ }^{160} \mathrm{Yb}$ & ${ }^{60} \mathrm{Ni}+{ }^{100} \mathrm{Mo}$ & $95-249$ & {$[31]$} & & 10 \\
${ }^{168} \mathrm{Yb}$ & ${ }^{18} \mathrm{O}+{ }^{150} \mathrm{Sm}$ & $63-126$ & {$[31,32]$} & {$[31,32]$} & 4 \\
${ }^{178} \mathrm{~W}$ & ${ }^{19} \mathrm{~F}+{ }^{159} \mathrm{~W}$ & $54-95$ & {$[31]$} & {$[31]$} & 4.2 \\
${ }^{188} \mathrm{Pt}$ & ${ }^{19} \mathrm{~F}+{ }^{169} \mathrm{Tm}$ & $50-91$ & {$[31]$} & {$[31]$} & 4.4 \\
${ }^{193} \mathrm{Tl}$ & ${ }^{28} \mathrm{Si}+{ }^{165} \mathrm{Ho}$ & $65-249$ & {$[33]$} & & 10 \\
${ }^{200} \mathrm{~Pb}$ & ${ }^{19} \mathrm{~F}+{ }^{181} \mathrm{Ta}$ & $49-153$ & {$[34,35]$} & {$[34,35]$} & 4.7 \\
${ }^{200} \mathrm{~Pb}$ & ${ }^{30} \mathrm{Si}+{ }^{170} \mathrm{Er}$ & $48-84$ & {$[34]$} & {$[34]$} & 10 \\
${ }^{216} \mathrm{Th}$ & ${ }^{32} \mathrm{~S}+{ }^{184} \mathrm{~W}$ & $125-203$ & {$[10]$} & {$[36]$} & 10 \\
${ }^{216} \mathrm{Ra}$ & ${ }^{19} \mathrm{~F}+{ }^{197} \mathrm{Au}$ & $39-86$ & {$[37]$} & {$[37]$} & 3 \\
${ }^{216} \mathrm{Ra}$ & ${ }^{30} \mathrm{Si}+{ }^{186} \mathrm{~W}$ & $39-83$ & {$[37]$} & {$[37]$} & 10 \\
${ }^{224} \mathrm{Th}$ & ${ }^{16} \mathrm{O}+{ }^{208} \mathrm{~Pb}$ & $26-83$ & {$[38]$} & {$[39,40]$} & 4 \\
\hline \hline
\end{tabular}


For the ${ }^{28} \mathrm{Si}+{ }^{165} \mathrm{Ho}$ and ${ }^{60} \mathrm{Ni}+{ }^{100} \mathrm{Mo}$ reactions, only evaporation residue data were measured. However, for these reactions, the $J_{0}$ values associated with fusion-like reactions are very high and the higher $J$ values all go into fission. The evaporation residue yield is therefore not sensitive to $J_{0}$ and is entirely determined by fission competition at lower $J$ values. Blann et al. [41] termed this a saturation analysis, as the higher $J$ values are saturated by fission.

For spallation reactions, we focused our efforts on proton-induced fission reactions of ${ }^{197} \mathrm{Au}$ [42], ${ }^{208} \mathrm{~Pb}$ [43], and ${ }^{238} \mathrm{U}$ [44] at $1 \mathrm{GeV}$, measured in inverse kinematics with the Fragment Separator (FRS) at SIS-GSI, Darmstadt, Germany. An additional experimental data set for $p+{ }^{208} \mathrm{~Pb}$ at $500 \mathrm{MeV}$ exists [45], but new measurements seem to indicate that the fission cross section was overestimated by about a factor of 2 [46]. We decided to normalize Fernandez et al.'s total fission cross section to the cross section measured by the CHARMS Collaboration, assuming that the fission distribution had been correctly measured. The reader should, nevertheless, keep in mind the normalization uncertainties associated with this data set.

The simultaneous-fitting strategy, however, cannot be applied to prescission neutron multiplicities, as there are no such data (to our knowledge) for spallation reactions. Moreover, accurate modeling of the pre- and postscission neutron data is likely to introduce new ingredients and parameters related to the the saddle-to-scission descent (e.g., the viscosity of the saddle-to-scission motion). It is not obvious whether the extra constraint provided by the new data would overcompensate for the increase in the number of degrees of freedom and lead to an effective decrease in the model uncertainty, all the more so because of the lack of relevant spallation data. Thus, we do not discuss prescission neutron multiplicities in this work.

\section{A. Modifications of the fission width}

The Bohr-Wheeler fission width,

$$
\Gamma_{\mathrm{BW}}=\frac{1}{2 \pi \rho_{\mathrm{n}}\left(E^{*}, J\right)} \int \mathrm{d} \epsilon \rho_{\mathrm{f}}\left[E^{*}-B(J)-\epsilon, J\right],
$$

is sensitive to the choice of the fission barrier $B$ and to the level-density parameters $a_{\mathrm{f}}$ and $a_{\mathrm{n}}$ associated with the saddlepoint and ground-state configurations. The $U$ dependence of the level-density parameter was initially assumed to be identical for the ground state and the saddle point, and it is described by Eq. (2). However, the saddle-point level-density parameter $a_{\mathrm{f}}$ was scaled by a constant factor with respect to the corresponding ground-state level-density parameter $a_{\mathrm{n}}$, to account for the increased surface area of the saddle-point configuration [47]. In the following, we refer to the scaling factor as "the $a_{\mathrm{f}} / a_{\mathrm{n}}$ ratio," for simplicity.

A number of modifications to the Bohr-Wheeler width have been proposed. In a one-dimensional derivation of the escape rate over a parabolic barrier for high viscosity, Kramers [48] obtained

$$
\Gamma_{K}=\left[\sqrt{1+\left(\frac{\gamma}{2 \omega}\right)^{2}}-\frac{\gamma}{\omega}\right] \Gamma_{\mathrm{BW}},
$$

where $\gamma$ is the magnitude of the viscosity, $\omega$ is the frequency associated with the inverted parabolic barrier, and the factor scaling the Bohr-Wheeler decay width is less than unity. Now $\omega$ is not expected to be a strong function of mass or spin, and if $\gamma$ is also constant, then the Kramers and the Bohr-Wheeler values differ by approximately a constant scaling factor. For this reason we have allowed a constant scaling to the Bohr-Wheeler width.

Lestone [49] developed a treatment of fission that explicitly included the tilting collective degree of freedom at the saddle point. Tilting is when the compound nucleus's spin is not perpendicular to the symmetry axis. For strongly deformed objects like the saddle-point configuration, this costs energy and thus decreases the fission probability. The decay width becomes

$$
\Gamma_{\text {Lestone }}=\Gamma_{\mathrm{BW}} \frac{\sum_{K=-J}^{J} \exp \left(-\frac{K^{2}}{2 I_{\text {eff }}}\right)}{2 J+1},
$$

where the summation is over $K$, the projection of the spin on the symmetry axis, and

$$
\frac{1}{I_{\text {eff }}}=\frac{1}{I_{\|}}-\frac{1}{I_{\perp}}
$$

with $I_{\|}$and $I_{\perp}$ the saddle-point moments of inertia parallel and perpendicular to the symmetry axis, respectively. In this work, the moments of inertia as well as the spin-dependent saddlepoint energies were taken from the finite-range calculations of Sierk [25]. Deviations from the Bohr-Wheeler value are largest for the highest spins, and thus the Lestone modification will be more important in fusion reactions.

We have tried to reproduce simultaneously the fission cross sections from fusion and spallation experiments using the following procedures.

(i) Adding a constant to the Sierk fission barriers for all spins.

(ii) Scaling the decay width by a constant factor.

(iii) Adjusting the $a_{\mathrm{f}} / a_{\mathrm{n}}$ ratio.

(iv) Using either the Bohr-Wheeler or the Lestone formalism.

(v) Introducing a constant fission delay.

\section{B. Fission probability}

Examples of fits to the ${ }^{19} \mathrm{~F}+{ }^{181} \mathrm{Ta} \rightarrow{ }^{200} \mathrm{~Pb}$ fission and evaporation-residue excitation functions are shown in Fig. 2. As the sum of these quantities (the fusion cross section) is fixed in the calculations, the degree to which the fission probability is reproduced is best gauged by the fit to the smaller quantity, that is, $\sigma_{\text {fis }}$ at low bombarding energies and $\sigma_{\mathrm{ER}}$ at higher values. Good fits were obtained with $\Gamma_{\mathrm{BW}} \times 2.46, a_{\mathrm{f}} / a_{\mathrm{n}}=1.00$ (longdashed curves), $\Gamma_{\mathrm{BW}} \times 1.00, a_{\mathrm{f}} / a_{\mathrm{n}}=1.036$ (solid curves), $\Gamma_{\text {Lestone }} \times 7.38, a_{\mathrm{f}} / a_{\mathrm{n}}=1.00$ (dotted curves), and $\Gamma_{\text {Lestone }} \times$ $1.00, a_{\mathrm{f}} / a_{\mathrm{n}}=1.057$ (short-dashed curves). The $\Gamma_{\mathrm{BW}} \times 2.46$, $a_{\mathrm{f}} / a_{\mathrm{n}}=1.00$ calculation is also almost identical to a $\Gamma_{\mathrm{BW}} \times$ $1.00, a_{\mathrm{f}} / a_{\mathrm{n}}=1.00$ calculation (not plotted) obtained with the Sierk fission barrier reduced by $1.0 \mathrm{MeV}$. With an even larger barrier reduction factor, one could arrive at a solution where 


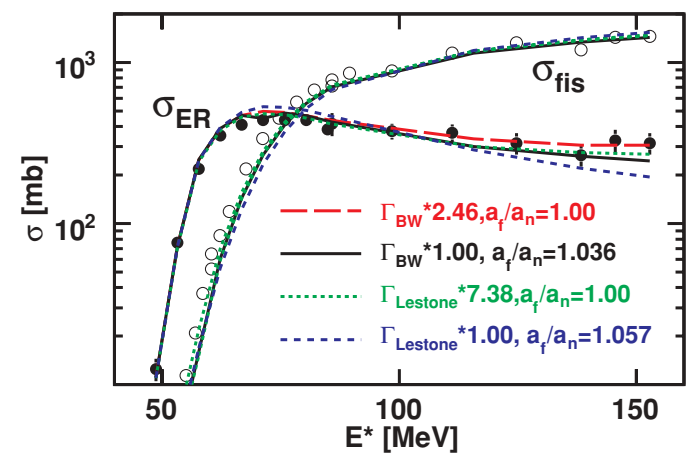

FIG. 2. (Color online) Comparison of GEMINI++ predictions to the experimental evaporation-residue (ER) and fission (fis) excitation functions for the ${ }^{19} \mathrm{~F}+{ }^{181} \mathrm{Ta}$ reaction.

the decay-width scaling factor is less than unity and consistent with the Kramers' scaling factor in Eq. (3).

As it is impossible to distinguish these different ways of modifying the fission probability from the fusion data alone, we now consider the constraint of adding the spallation data to the analysis. In Fig. 3, we show the equivalent calculations for the mass distributions of the products of the $1-\mathrm{GeV} p+{ }^{208} \mathrm{~Pb}$ spallation reaction. Of all these possibilities, the $\Gamma_{\mathrm{BW}} \times 1.00$, $a_{\mathrm{f}} / a_{\mathrm{n}}=1.036$ calculation best reproduces the yield of the fission peak. This highlights the significant reduction in fittingparameter ambiguity that can be obtained by simultaneously fitting heavy-ion and spallation data. We also note that the $\Gamma_{\mathrm{BW}} \times 2.46, a_{\mathrm{f}} / a_{\mathrm{n}}=1.00$ calculation and the reduced fission barrier calculation with $\Gamma_{\mathrm{BW}} \times 1.00, a_{\mathrm{f}} / a_{\mathrm{n}}=1.00$ (not shown) were again identical. Thus, while many of the ambiguities in the fitting parameters have been removed, the ambiguity between the effect of the magnitude of the fission barrier and the Kramers scaling factor was not eliminated with simultaneous fitting. We will continue using the $\Gamma_{\mathrm{BW}} \times 1.00$, $a_{\mathrm{f}} / a_{\mathrm{n}}=1.036$ calculation as our best fit to both sets of experimental data, but it should be noted that, with reduced fission barriers, an equivalent solution with a Kramers scaling factor $(<1)$ can also be obtained. We are just not able to constrain the magnitude of the Kramers factor from all these data.

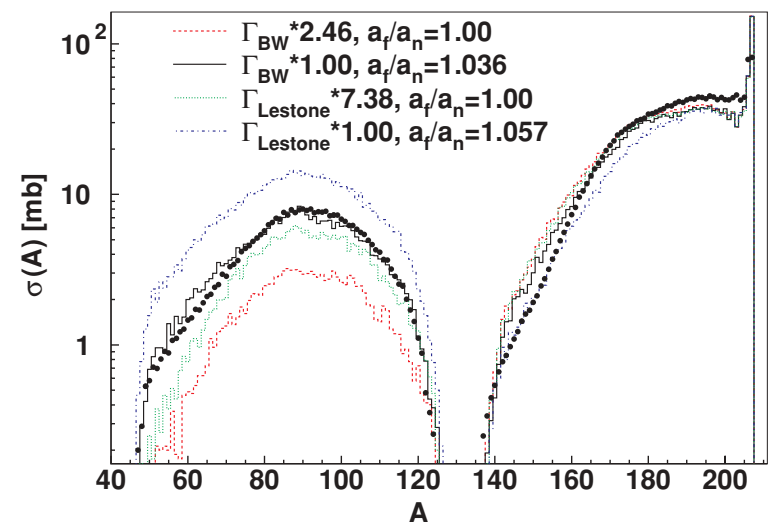

FIG. 3. (Color online) Comparison of experimental and calculated residual mass distributions for the $1-\mathrm{GeV} p+{ }^{208} \mathrm{~Pb}$ reaction. Predictions of the INCL4.5-GEMINI++ code are shown for different adjustments of the fission width. Experimental data are from Ref. [43].

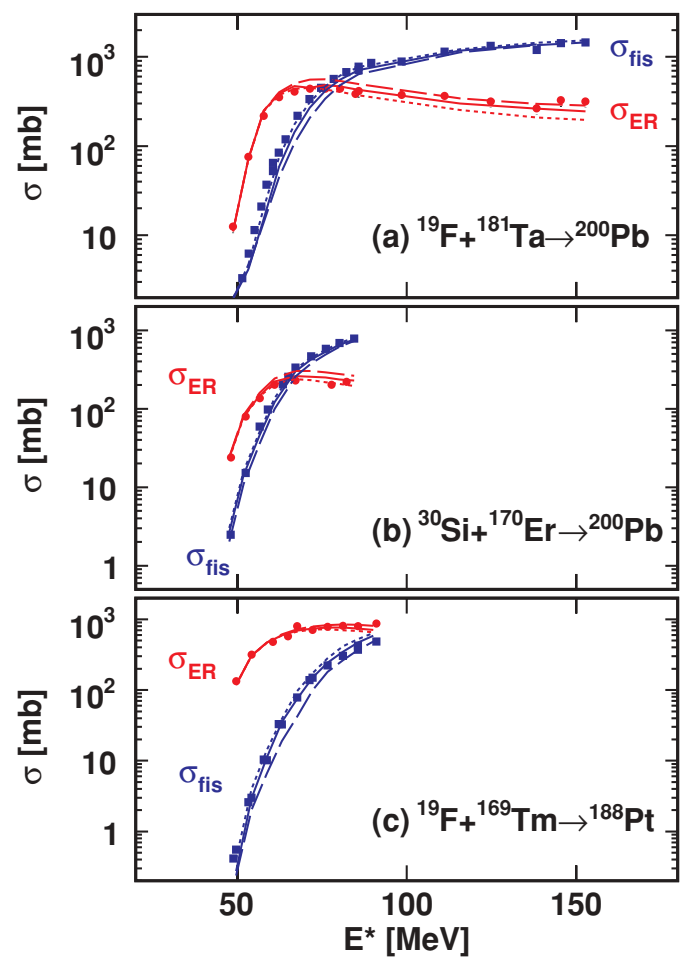

FIG. 4. (Color online) Comparison of experimental and calculated fission (fis) and evaporation-residue (ER) excitation functions for the indicated reactions. Solid lines: Bohr-Wheeler fission width, $a_{\mathrm{f}} / a_{\mathrm{n}}=1.036$, no fission delay. Dashed lines: Lestone fission width, $a_{\mathrm{f}} / a_{\mathrm{n}}=1.057,1$-zs fission delay. Dotted lines: Bohr-Wheeler fission width, energy-dependent effective $a_{\mathrm{f}} / a_{\mathrm{n}}$ ratio with $r=1.0747$.

Comparisons of GEMINI++ predictions to the experimental fission and evaporation-residue excitation functions listed for the heavy-ion-induced fusion reactions in Table II are shown in Figs. 4 to 7. Solid curves show the predictions with $a_{\mathrm{f}} / a_{\mathrm{n}}=1.036$, Sierk fission barriers, and no scaling of the Bohr-Wheeler decay width. For spallation, Figs. 8 to 10 show comparisons between measured and calculated residue mass distributions. Finally, Fig. 11 shows a comparison between measured [50] and calculated excitation curves for the fission cross section in proton collisions with ${ }^{181} \mathrm{Ta}$, a low-fissility target.

The central result is that it is possible to reproduce the total fission cross section for all the studied spallation reactions by adjusting only one free parameter, namely, the $a_{\mathrm{f}} / a_{\mathrm{n}}$ ratio, which was set equal to 1.036 in our calculations, while the global scaling of the fission width and of the fission barrier were kept equal to 1; no Lestone correction was introduced. A global scaling of the fission width is roughly equivalent to a reduction of the barrier height, but in both cases, these adjustments alone do not fit the data. The adjustment of the $a_{\mathrm{f}} / a_{\mathrm{n}}$ ratio, in contrast, is characterized by a different excitation-energy dependence, which is better suited for the description of fission from spallation remnants. The Lestone correction, which suppresses the fission width at high spin, does not have a large effect on spallation data, some $80 \%$ at most. This is caused by the low angular momenta generated in the intranuclear cascade. 


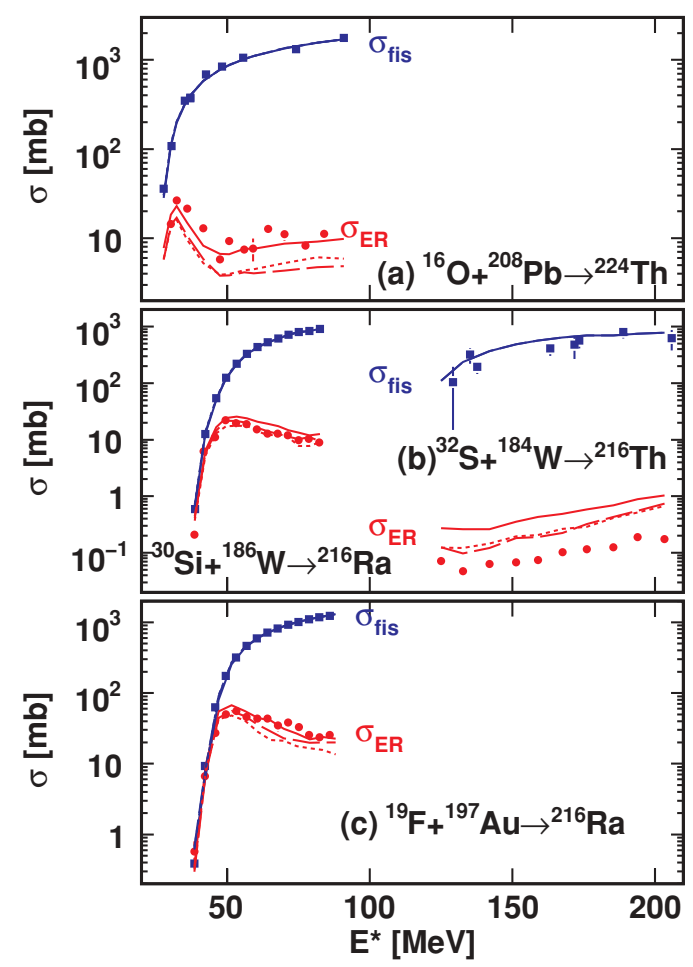

FIG. 5. (Color online) Same as Fig. 4.

For the heavy-ion-induced fusion data, the GEMINI++ predictions are also generally quite good, however, there are a couple of reactions for which significant deviations are found. First, for the ${ }^{18} \mathrm{O}+{ }^{150} \mathrm{Sm} \rightarrow{ }^{168} \mathrm{Yb}$ and ${ }^{19} \mathrm{~F}+{ }^{139} \mathrm{La} \rightarrow{ }^{158} \mathrm{Dy}$ reactions shown in Figs. 6(b) and 6(c), the fission cross section is overestimated by almost an order of magnitude. In compar-

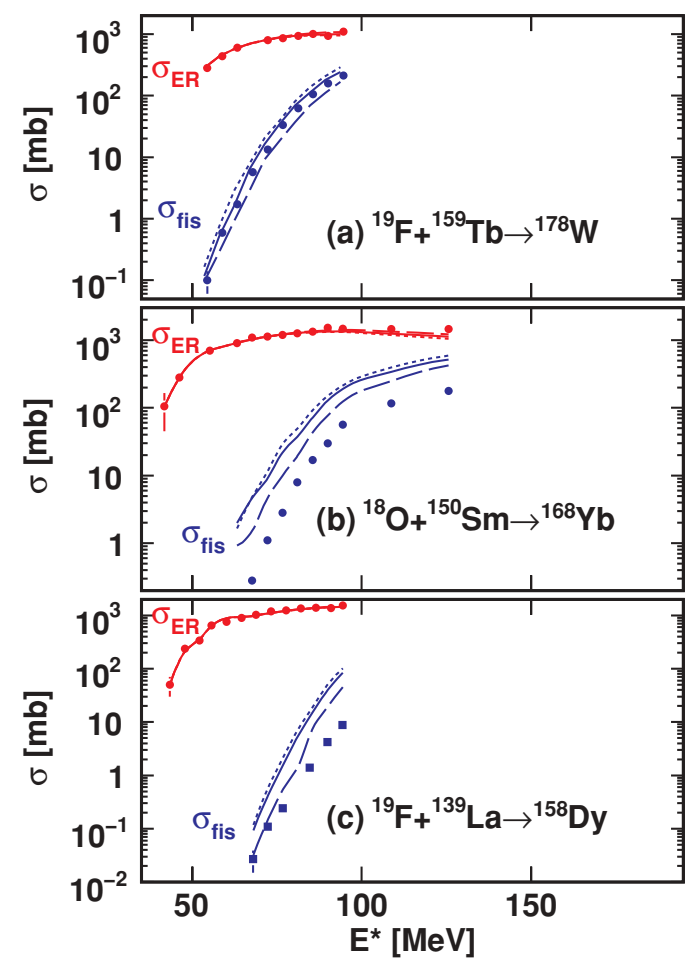

FIG. 6. (Color online) Same as Fig. 4.

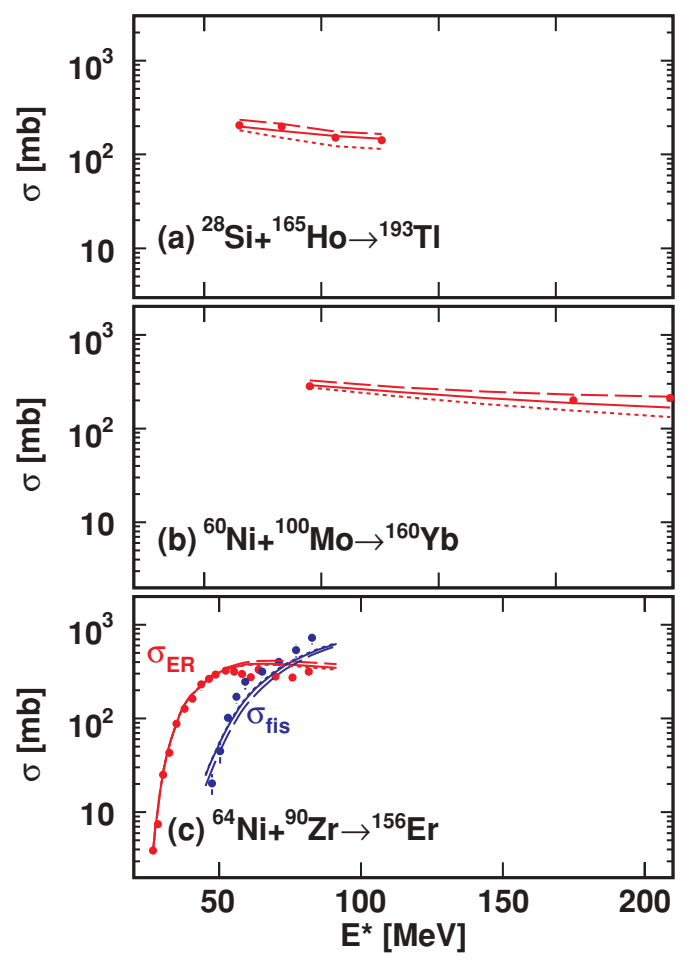

FIG. 7. (Color online) Same as Fig. 4.

ison, the Ni-induced reactions in Figs. 7(b) and 7(c) making similar-mass compound nuclei are reproduced much better. It is difficult to understand these ${ }^{18} \mathrm{O}$ - and ${ }^{19} \mathrm{~F}$-induced reactions, and previous attempts also failed to reproduce the data [26]. For instance, at the highest bombarding energies, the excitation energies probed in these reactions overlap those in the $\mathrm{Ni}$ induced reactions. Similarly, the predicted angular-momentum region over which the fission yield is determined in the $\mathrm{O}$ - and F-induced reactions is similar to that in which the residue yield is determined in Ni-induced reactions. Unless there are significant nonfusion processes, such as pre-equilibrium or incomplete fusion, occurring, these data suggest an entrancechannel dependence of the fission decay probability, which would violate the compound-nucleus hypothesis. However,

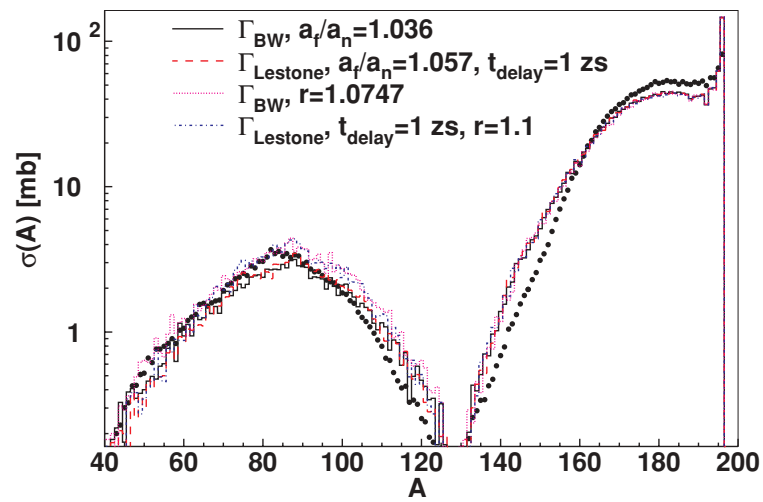

FIG. 8. (Color online) Residue-mass distribution for the $p+{ }^{197} \mathrm{Au}$ reaction at $1 \mathrm{GeV}$. Predictions of the INCL4.5-GEMINI++ code are shown for different adjustments of the fission width. Experimental data are from Ref. [42]. 


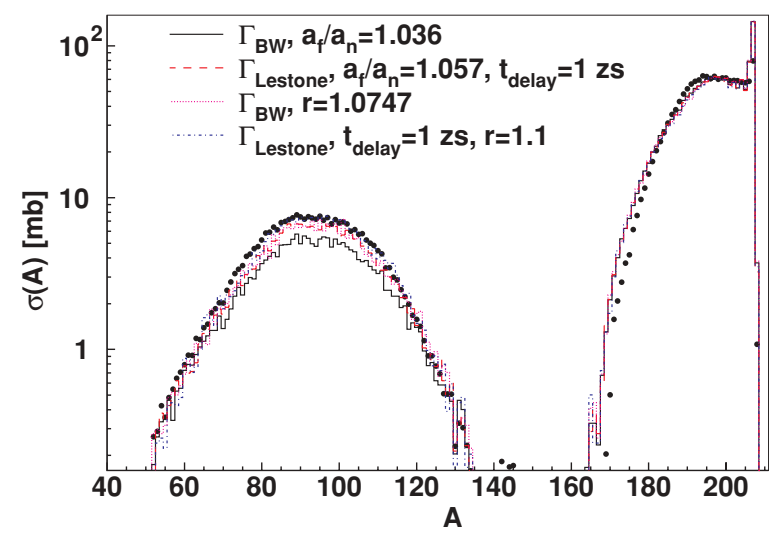

FIG. 9. Same as Fig. 8, but for $p+{ }^{208} \mathrm{~Pb}$ at $500 \mathrm{MeV}$. Experimental data from Refs. [45] and [51]. Experimental fission cross sections have been multiplied by a factor of 146/232 (see text for details).

we see no evidence of such an effect for the O- and F-induced reactions with heavier targets. Clearly, our understanding of fission for $A<170$ is lacking and more studies are needed.

The other case where the GEMINI++ predictions fail is for the ${ }^{32} \mathrm{~S}+{ }^{184} \mathrm{~W} \rightarrow{ }^{216} \mathrm{Th}$ reaction in Fig. 5(a). Here the evaporation-residue cross section is exceedingly small $(\sim 0.1 \mathrm{mb})$ and is overpredicted by almost an order of magnitude. However, the calculations get the excitation-energy dependence of the cross sections correct, which previous calculations could not do without invoking an excitationenergy dependence of the dissipation strength [10]. In our calculations the predicted excitation-energy dependence of the residue cross section is a consequence of the assumed excitation-energy dependence of the level-density parameter [5]. Low-probability events in the statistical model are generally quite sensitive to the statistical-model parameters. In this case, it was demonstrated that the residue yield is very sensitive to the absolute value of the level-density parameter and its excitation-energy dependence [5]. For example, the residue yield is increased by $2-3$ orders of magnitude when the level-density parameter is changed from $a=A / 7.3$ to $A / 11 \mathrm{MeV}^{-1}$. Further refinement of the value of this parameter at the higher excitation energies probed in this more symmetric fusion reaction may be needed in the future.

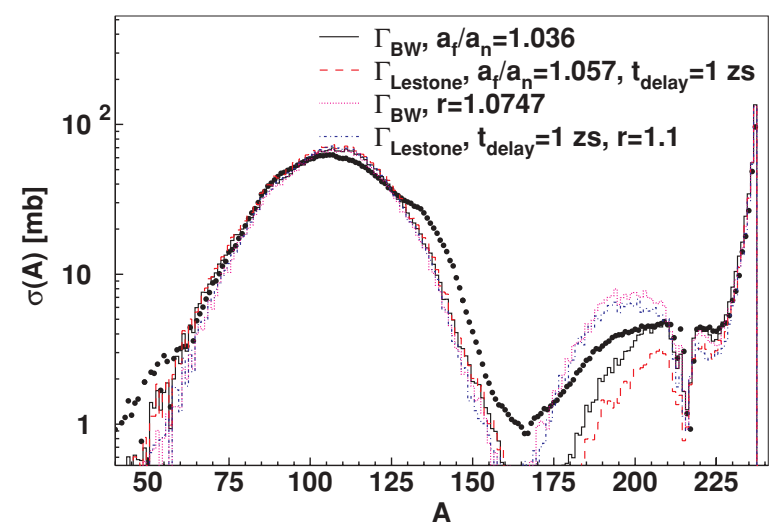

FIG. 10. (Color online) Same as Fig. 8, but for $p+{ }^{238} \mathrm{U}$ at $1 \mathrm{GeV}$. Experimental data are from Refs. [44], [52], and [53].

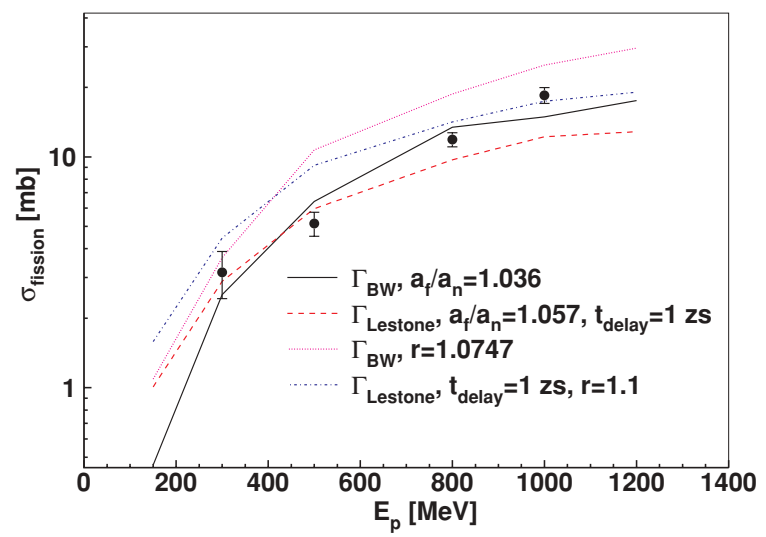

FIG. 11. (Color online) Excitation curve for the fission cross section in $p+{ }^{181} \mathrm{Ta}$. Predictions of the INCL4.5-GEMINI++ code are shown for different adjustments of the fission width. Experimental data are from Ref. [50].

Alternatively, there is evidence that for this mass region, quasifission completes with fusion reactions even at the lower $\ell$ waves associated with evaporation-residue production [37,54]. In the case of the ${ }^{216} \mathrm{Ra}$ compound nucleus in Figs. 5(b) and 5(c), Berriman et al. have indicated that both the ${ }^{19} \mathrm{~F}+{ }^{197} \mathrm{Au}$ and the ${ }^{30} \mathrm{Si}+{ }^{184} \mathrm{~W}$ reactions have reduced evaporation-residue cross sections, owing to quasifission competition [37]. Therefore this higher-mass region for heavy-ion reactions is subject to more uncertainty in constraining the statistical-model parameters.

\section{Fission-fragment mass distributions}

Previous treatments of the fission-fragment mass distribution have assumed thermal models where the mass division is determined either at the saddle point (Moretto's formalism) or at the scission configuration [55]. In reality, a complete description probably requires one to follow the trajectory from saddle to scission including fluctuations, for example, by Langevin simulations [56,57]. However for large-scale simulations, this is too time-consuming, so a simpler and faster procedure for determining mass division is required.

Experimental mass distributions for heavy-ion-induced fusion-fission reactions are shown in Figs. 12 and 13. In these figures, the experimental fission-fragment mass was not directly measured, but rather the ratio of primary masses (before postscission particle evaporation) was inferred from either the ratio of the measured fission-fragment velocities or the kinetic energies. The absolute primary mass was assumed to be equal to the compound-nucleus mass, which of course ignores the prescission evaporation of light particles. However, the distributions simulated by GEMINI++ (curves in Figs. 12 and 13) were analyzed in the same manner as the experimental data and thus contain the same deficiencies.

The data in Fig. 12 are for the ${ }^{16} \mathrm{O}+{ }^{182} \mathrm{~W} \rightarrow{ }^{198} \mathrm{~Pb}$ reactions. The relative mass distributions were obtained from Ref. [58] and absolute normalization was achieved using the fission cross sections measured in Ref. [59]. The data in Fig. 13 are for the ${ }^{216} \mathrm{Ra}$ compound nucleus at $E^{*} \sim 60 \mathrm{MeV}$ with three entrance channels: ${ }^{12} \mathrm{C}+{ }^{204} \mathrm{~Pb},{ }^{19} \mathrm{~F}+{ }^{197} \mathrm{Au}$, and 


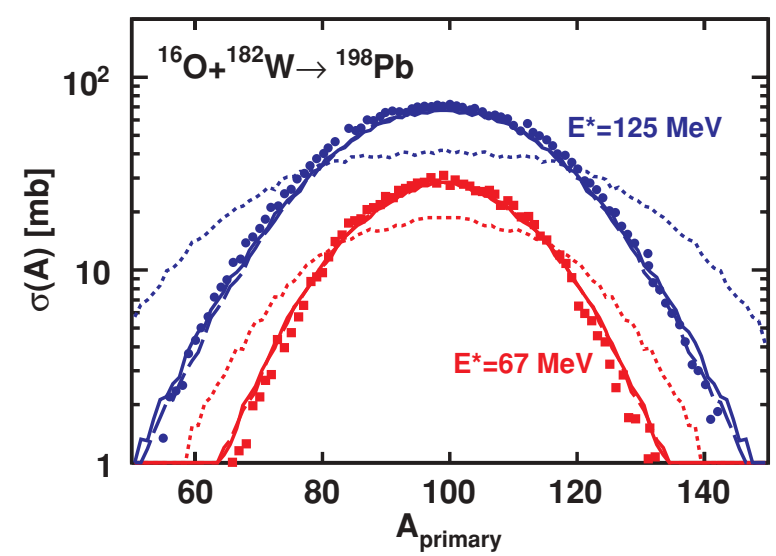

FIG. 12. (Color online) Comparison of experimental (data points) and simulated (curves) distributions of the primary fission-fragment masses for the ${ }^{198} \mathrm{~Pb}$ compound nucleus formed in the ${ }^{16} \mathrm{O}+{ }^{182} \mathrm{~W}$ reaction at the two indicated excitation energies. The total fission cross section has been calculated using the Bohr-Wheeler formalism, $a_{\mathrm{f}} / a_{\mathrm{n}}=1.036$, without any fission delay. The dotted curve was obtained using the Moretto formalism with Sierk's conditional barriers to define the mass distributions. Solid and dashed curves were obtained using the Rusanov systematics with the saddle- and scission-point temperatures, respectively.

${ }^{28} \mathrm{Si}+{ }^{186} \mathrm{~W}$. The fission excitation function for the latter two are shown in Figs. 5(b) and 5(c).

In Fig. 12, the dotted curves show the mass distribution determined from Moretto's formalism using interpolated values of Sierk's finite-range calculations for the conditional barriers [14]. The total fission width (the total width for all mass divisions associated with the peak in the mass distribution) was normalized to the Bohr-Wheeler value for these calculations. Therefore in this figure, only the shape of the mass distribution is determined from the Moretto formalism. Clearly, these distributions are much wider than the experi-

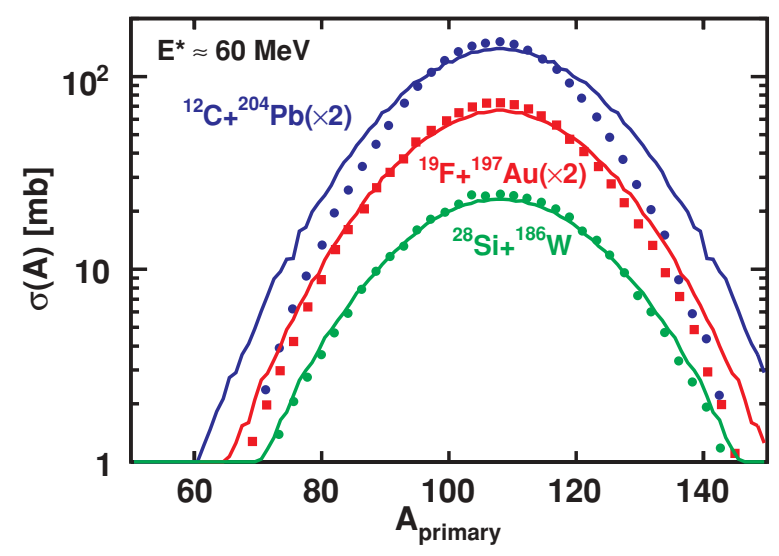

FIG. 13. (Color online) Comparison of experimental (data points) and simulated (curves) distributions of the primary fission-fragment masses for the ${ }^{216} \mathrm{Ra}$ compound nucleus at $E^{*} \sim 60 \mathrm{MeV}$ formed in the ${ }^{12} \mathrm{C}+{ }^{204} \mathrm{~Pb},{ }^{19} \mathrm{~F}+{ }^{197} \mathrm{Au}$, and ${ }^{30} \mathrm{Si}+{ }^{186} \mathrm{~W}$ reactions. The total fission cross section has been calculated using the Bohr-Wheeler formalism, $a_{\mathrm{f}} / a_{\mathrm{n}}=1.036$, without any fission delay. To aid viewing, the data and curves have been scaled by the indicated amounts. mental quantities. This is quite typical of other cases where a peak exists in the mass distribution at symmetry. See, for example, the study of ${ }^{151} \mathrm{Eu}$ compound nuclei in Ref. [60] and the light, but high-spin ${ }^{110} \mathrm{Sn}$ compound nuclei studied in Ref. [61]. For less fissile nuclei, where the mass distribution has a minimum at symmetric division, the Moretto formalism (with Sierk barriers) gives a much better description of the experimental data. See, for example, the studies of ${ }^{111}$ In [18], ${ }^{102} \mathrm{Rh}$ and ${ }^{105} \mathrm{Ag}$ [16], and ${ }^{75} \mathrm{Br}$ [20] compound nuclei.

The cause of this inadequacy for heavier systems could either be an incorrect asymmetry dependence of Sierk's conditional barriers or a failure of Moretto's formalism. The latter predicts the asymmetry distribution at the ridge line of conditional saddle points and assumes that the mass asymmetry is unchanged during the descent from saddle to scission.

As an alternative to using Moretto's formalism, we used the systematics of fission-fragment mass distributions complied by Rusanov et al. [21]. The mass distribution is assumed to be Gaussian and its variance is parameterized as

$$
\sigma_{A}^{2}=\frac{A_{\mathrm{CN}}^{2} T}{16 \frac{d^{2} V}{d \eta^{2}}\left(Z^{2} / A, J\right)},
$$

where $\frac{d^{2} V}{d \eta^{2}}$ is the second derivative of the potential energy surface with respect to the mass-asymmetry deformation parameter $\left(\eta=2 \frac{A_{1}-A_{2}}{A_{1}+A_{2}}\right.$, where $A_{1}$ and $A_{2}$ is the mass division). This quantity is parameterized as a function of the fissility $Z^{2} / A$ and the spin $J$. The quantity $T$ is the nuclear temperature, where

$$
\frac{1}{T}=\frac{d \ln \rho}{d U} .
$$

Rusanov et al. considered three parametrizations of $\frac{d^{2} V}{d \eta^{2}}$, with three different temperatures, as follows: (i) the temperature of the fission nucleus at the saddle point is used, but no presaddle light-particle evaporations are allowed; (ii) as temperature i, but presaddle evaporations are allowed; or (iii) the temperature at the scission point is used. The first of these is not realistic and was not considered and the second is basically consistent with the ideas of the Moretto formalism. The latter two can be called saddle-point and scission-point models where the mass distributions are both determined thermally. In these two cases, the quantity $\frac{d^{2} V}{d \eta^{2}}$ should be identified with the asymmetry dependence of the potential-energy surface at the saddle and scission points, respectively.

In GEMINI++, once fission is decided for an event, evaporation during the saddle-to-scission transition is allowed. This is important for the scission model, as we need to determine the temperature at the scission point. The saddle-to-scission evaporation is treated in a simplified manner using spherical level densities and transmission coefficients in the Weisskopf-Ewing evaporation formalism with the deformation-plus-rotational energy removed from the total excitation energy. The deformation-plus-rotational energy of the scission configuration is determined as the sum of fissionfragment kinetic energy from Viola's systematics [62] and the fission $Q$ value. Evaporation during the saddle-to-scission 


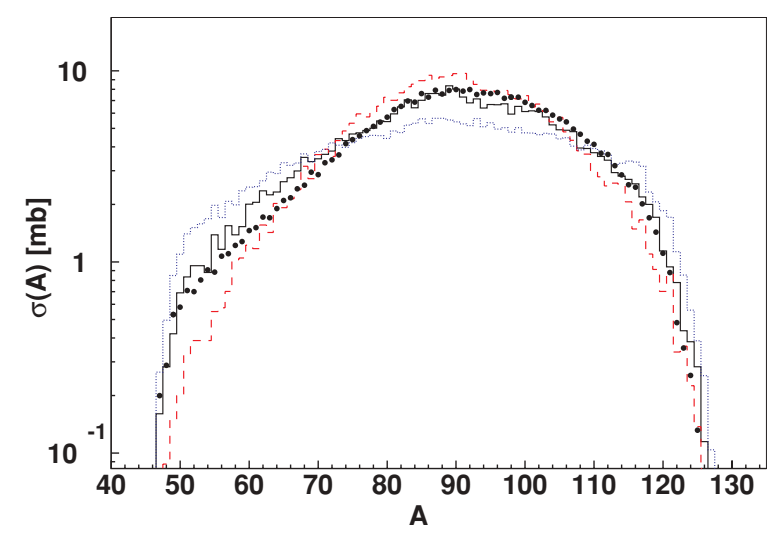

FIG. 14. (Color online) Comparison of experimental (data points) and simulated (curves) distributions of the fission-fragment masses for the $1-\mathrm{GeV} p+\mathrm{Pb}$ spallation reaction. The dotted (blue) curve was obtained using the Moretto formalism with Sierk's conditional barriers to define the mass distributions. The solid (black) and dashed (red) curves were obtained using the Rusanov systematics with the saddle- and scission-point temperatures, respectively.

transition occurs for a period proportional to the difference in energy between the saddle and the scission points, that is,

$$
t_{\mathrm{ss}}=k_{\mathrm{ss}}\left(E_{\mathrm{saddle}}-E_{\mathrm{scission}}\right),
$$

consistent with a high viscosity. The parameter $k_{\mathrm{ss}}$, which is related to the magnitude of this viscosity, was fixed to $k_{\mathrm{ss}}=$ $1 \mathrm{zs} / \mathrm{MeV}$ by fitting prescission neutron multiplicities from Ref. [7].

The solid and dashed curves in Fig. 12 show the predictions with the Rusanov saddle- and scission-point systematics, respectively. These predictions are almost identical, and for the lowest excitation energy, the curves completely overlap and cannot be distinguished. This is not surprising, as both Rusanov systematics are fits to ${ }^{3} \mathrm{He}$-induced fission and fusion-fission data including the data set in Fig. 12. However, at higher excitation energies such as those sampled in spallation reactions, the two systematics give quite different predictions, as the thermal excitation at scission increases much more slowly with compound-nucleus excitation than does the saddle-point value [7]. Figure 14 compares the two systematics for the $1-\mathrm{GeV} p+\mathrm{Pb}$ spallation reaction. In this case, the predicted mass distribution obtained with the scission systematics (dashed curve) is too narrow, while the saddle systematics (solid curve) gives a good agreement.

The success of Rusanov's saddle-point systematics thus suggests that the fission mass division is determined quite close to the saddle-point configuration. It addition, it indicates that the Moretto formalism is still applicable for near-symmetric divisions of heavy nuclei. However, it should not be used with Sierk's conditional barriers in this region.

The differences between the mass-asymmetry dependence of Sierk's conditional barriers and the Rusanov systematics are shown directly in Fig. 15 for ${ }^{149} \mathrm{~Tb}$ and ${ }^{194} \mathrm{Hg}$ compound nuclei at $J=0$. Dashed curves are parabolic functions with curvatures from the Rusanov systematics and with symmetric fission barriers from Sierk's calculations. The Rusanov results have larger curvatures at symmetry than Sierk's predictions and thus

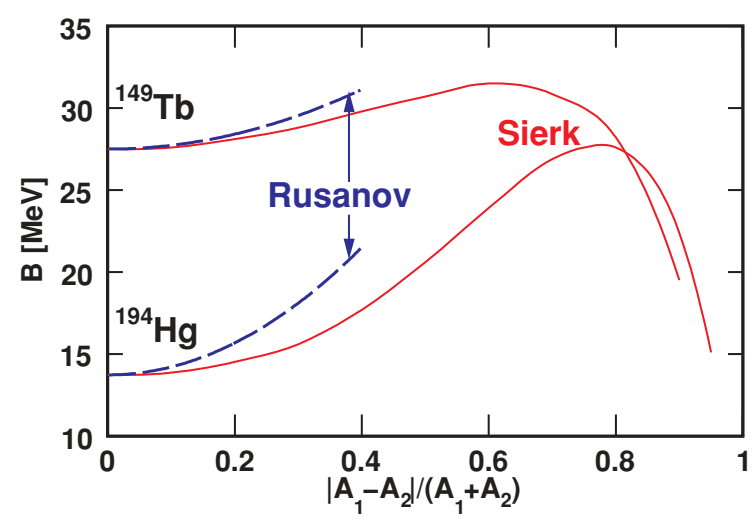

FIG. 15. (Color online) Comparison of asymmetry dependences of conditional barriers for ${ }^{149} \mathrm{~Tb}$ and ${ }^{194} \mathrm{Hg}$ nuclei at $J=0$. Solid curves are predictions from Sierk's finite-range calculations. Dashed curves are parabolic functions with curvatures taken from the $\mathrm{Ru}$ sanov systematics and with symmetric fission barriers taken from the finite-range calculations. The mass-asymmetry coordinate is defined in terms of $A_{1}$ and $A_{2}$, the two masses following binary division.

give narrower fission-fragment mass distributions. The differences between Sierk's predictions and the Rusanov systematics is much larger for the heavier ${ }^{194} \mathrm{Hg}$ nucleus. For even heavier nuclei, the asymmetry coordinate in the finite-range calculations becomes undefined, as the saddle-point configuration has no well-defined neck [63]. If this is the case, then the Moretto formalism is no longer applicable for these systems and the mass asymmetry is determined during the descent from saddle to scission. In such cases the interpretation of the Rusanov systematics in terms of a Moretto type may be suspect. We note that the $Z^{2} / A$ dependence of $d^{2} V / d \eta^{2}$ in the Rusanov systematics has an abrupt slope change at $Z^{2} / A=24$, possibly related to this effect. However, even in the $p+\mathrm{U}$ spallation reaction we produce $Z^{2} / A$ ratios that are below this value.

The Rusanov saddle systematics was used for the other spallation predictions in Figs. 8 to 10 and gives quite good agreement. However, for the $p+{ }^{238} \mathrm{U}$ reaction in Fig. 10, the simulation fails to reproduce the small shoulder in the fission mass distribution for higher masses. The Rusanov systematics only gives the width of the distribution and will not predict finer structures linked to shell effects, such as these.

In Fig. 13, the simulated mass distributions (from the saddle systematics) for the ${ }^{216} \mathrm{Ra}$ compound nuclei reproduce the data reasonably well with the exception of the ${ }^{12} \mathrm{C}+{ }^{204} \mathrm{~Pb}$ data, where the experimental distribution is somewhat narrower. Berriman et al. [37] suggest that the ${ }^{12} \mathrm{C}+{ }^{204} \mathrm{~Pb}$ data are all fusion-fission, while the ${ }^{19} \mathrm{~F}+{ }^{197} \mathrm{Au}$ and ${ }^{30} \mathrm{Si}+{ }^{184} \mathrm{~W}$ data contain quasifission contributions, making the mass distributions wider. This would imply that for the more massive compound nucleus, the Rusanov systematics overestimate the width of the statistical fission mass distributions, as many of the heavy-ion data used in these systematics have contributions from quasifission. However, the Rusanov systematics also contains the lower-spin ${ }^{3} \mathrm{He}$-induced fission data in this mass region, and there quasifission is expected to be absent. Thus spallation mass distributions that sample lower spins are not expected to suffer from this problem. 


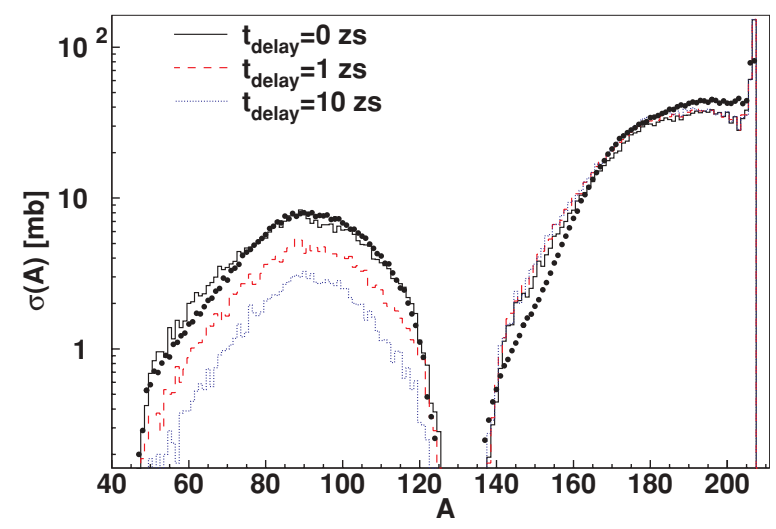

FIG. 16. (Color online) Same as Fig. 3, but for the Bohr-Wheeler fission width, $a_{\mathrm{f}} / a_{\mathrm{n}}=1.036$, and three values of fission delay.

\section{Fission delays}

Apart from the lighter compound nuclei, we have demonstrated that a unified description of fission widths in fusion and spallation reactions can be obtained. The solution is unique, apart from an ambiguity between the height of the fission barrier and the Kramers scaling factor. However, we now show that another ambiguity arises when fission transients are considered. To show the sensitivity of predictions to transients, we have incorporated a simple implementation of these in GEMINI++; the fission width is set to 0 for a time $t_{\text {delay }}$, after which it assumes its asymptotic value. During this fission-delay period, the compound nucleus can decay by light-particle evaporation and intermediate-mass-fragment emission. The fission delay is expected on theoretical grounds to be logarithmically dependent on the nuclear temperature [6], but this weak dependence (and any mass dependence) has been neglected in the first approximation. Figures 16 and 17 show the dependence of the predicted mass distributions for the $1-\mathrm{GeV} p+{ }^{208} \mathrm{~Pb}$ reaction with $t_{\text {delay }}=0,1$, and $10 \mathrm{zs}$. Even a short, 1-zs, delay has a large effect on the yield in the fission peak. Therefore, spallation reactions should be quite sensitive to the fission transients. Tishchenko et al. also expected large reductions in the fission probability in $2.5-\mathrm{GeV} p+{ }^{197} \mathrm{Au}$, ${ }^{209} \mathrm{Bi}$, and ${ }^{238} \mathrm{U}$ reactions owing to fission transients; however,

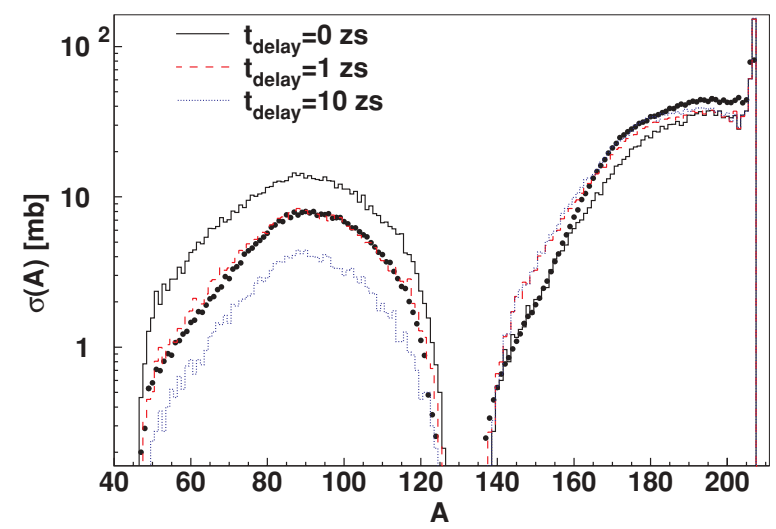

FIG. 17. (Color online) Same as Fig. 3, but for the Lestone fission width, $a_{\mathrm{f}} / a_{\mathrm{n}}=1.057$, and three values of fission delay.
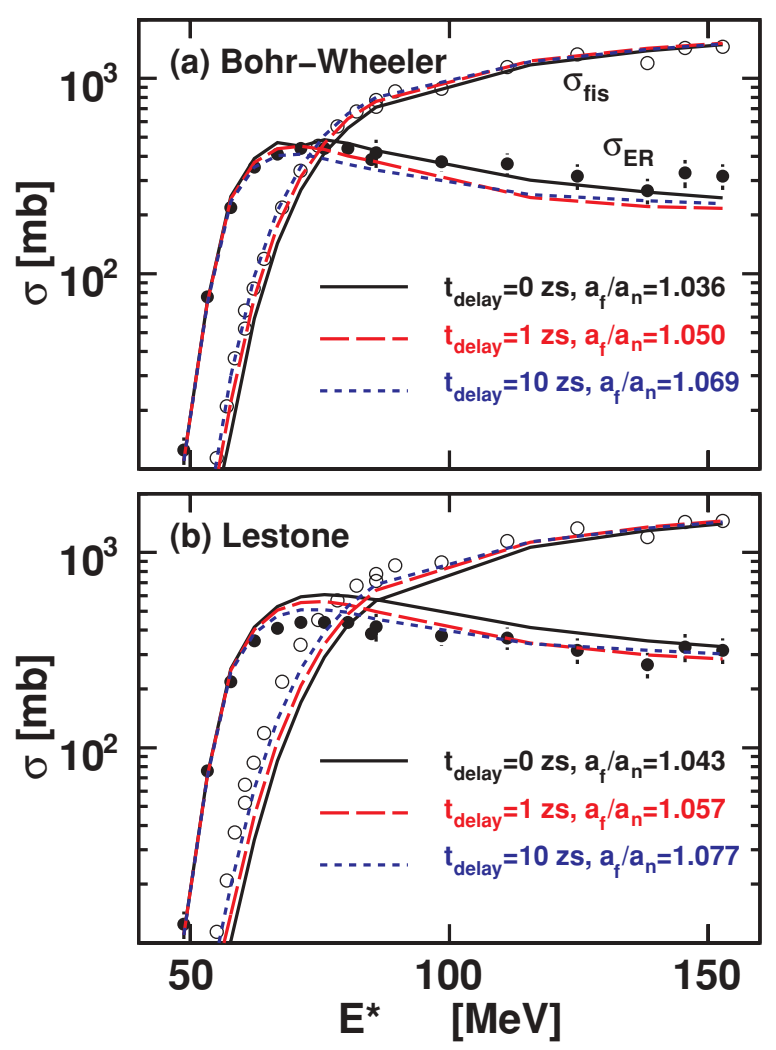

FIG. 18. (Color online) Comparison of GEMINI++ predictions using (a) the Bohr-Wheeler and (b) the Lestone fission formalism for the experimental evaporation-residue and fission excitation functions for the ${ }^{19} \mathrm{~F}+{ }^{181}$ Ta reaction. Curves are labeled by the $t_{\text {delay }}$ and $a_{\mathrm{f}} / a_{\mathrm{n}}$ values obtained from fitting the fission cross section for the $1-\mathrm{GeV}$ $p+\mathrm{Pb}$ reaction.

they were also able to reproduce the fission yield within the standard statistical-model framework [13].

Jing et al. [64] found that the effect of increasing the fission delay can be largely counteracted by increasing the value of the $a_{\mathrm{f}} / a_{\mathrm{n}}$ parameter. Both parameters have little effect on the fission probability at low excitation energies. However, with increasing excitation energy, the fission probability becomes ever more sensitive to both $t_{\text {delay }}$ and $a_{\mathrm{f}} / a_{\mathrm{n}}$. Even with the large range of excitation energies explored in this work, we found that it is impossible to break the ambiguity between $t_{\text {delay }}$ and $a_{\mathrm{f}} / a_{\mathrm{n}}$. To illustrate this, Fig. 18 compares the ${ }^{200} \mathrm{~Pb}$ fusion data to GEMINI++ calculations with fission delay for both the Bohr-Wheeler [Fig. 18(a)] and the Lestone [Fig. 18(b)] formalisms. The values of $t_{\text {delay }}$ and $a_{\mathrm{f}} / a_{\mathrm{n}}$ listed in these figures were obtained by reproducing the fission cross section in the $1-\mathrm{GeV} p+\mathrm{Pb}$ spallation reaction. For the Bohr-Wheeler case in Fig. 18(a), one see that the calculations with $t_{\text {delay }}=1$ and $10 \mathrm{zs}$ are almost identical and within $30 \%$ of the experimental values. The calculation with $t_{\text {delay }}=0 \mathrm{zs}$ fits the data somewhat better, but all calculations can be deemed acceptable.

For the Lestone formalism in Fig. 18(b), the inclusion of a delay with $t_{\text {delay }}>1 \mathrm{zs}$ improves the agreement with the data. As in Fig. 18(a), the calculations with $t_{\text {delay }} \geqslant 1 \mathrm{zs}$ are again almost identical. The Lestone prescription with fission delay also allows good agreement with the other data sets we have considered; see the dotted curves in Figs. 4 to 7 
(fusion) and Figs. 8 to 10 (spallation), which were obtained with $a_{\mathrm{f}} / a_{\mathrm{n}}=1.057$ and $t_{\text {delay }}=1 \mathrm{zs}$. Calculations with the larger $t_{\text {delay }}$ values produce a similar level of agreement. It is thus clear that the magnitude of the fission transients cannot be deduced from the fission probability alone.

\section{FISSION AT VERY HIGH EXCITATION ENERGIES}

Fission cross sections in fusion and spallation reactions are dominated by the most densely populated regions of the compound-nucleus $E^{*}-J$ plane (Fig. 1). The successful reproduction of these data thus indicates that the GEMINI++ model gives an efficient description of fission from compound nuclei with excitation energies up to $\sim 300 \mathrm{MeV}$ and spins up to $\sim 60 \hbar$.

It is possible to probe beyond this region if one considers other types of data. Tishchenko et al. [65] studied proton-induced spallation reactions at $2.5 \mathrm{GeV}$ on gold, bismuth, and uranium targets. They measured the fission probability in coincidence with the neutron, hydrogen, and helium multiplicities, which can be used to reconstruct the excitation energy after the intranuclear cascade. They were able to reproduce the measurements with an old version of the INCL-GEMINI model by tuning the value of $a_{\mathrm{f}} / a_{\mathrm{n}}$ on a system-by-system basis, ranging from 1.000 for the uranium target to 1.022 for the gold target. These $a_{\mathrm{f}} / a_{\mathrm{n}}$ values are smaller than those discussed in the present work.

Figure 19 indeed shows that our candidate parameter sets (Bohr-Wheeler, $a_{\mathrm{f}} / a_{\mathrm{n}}=1.036$; Lestone, $a_{\mathrm{f}} / a_{\mathrm{n}}=1.057$, $t_{\text {delay }}=1 \mathrm{zs}$ ) largely overestimate the fission probability at high excitation energies deduced by Tishchenko et al. for $p+\mathrm{U}$. Note that the fission probability is well reproduced up to a few hundred mega-electron volts, which is coherent with the results of the previous section. The shape of the curve is indeed very sensitive to the value of $a_{\mathrm{f}} / a_{\mathrm{n}}$, as Fig. 19 shows. We can interpret this result as an indication of the fact that while a large value of $a_{\mathrm{f}} / a_{\mathrm{n}}$ is appropriate at a low excitation energy, the effective $a_{\mathrm{f}} / a_{\mathrm{n}}$ value at a high excitation energy should be smaller.

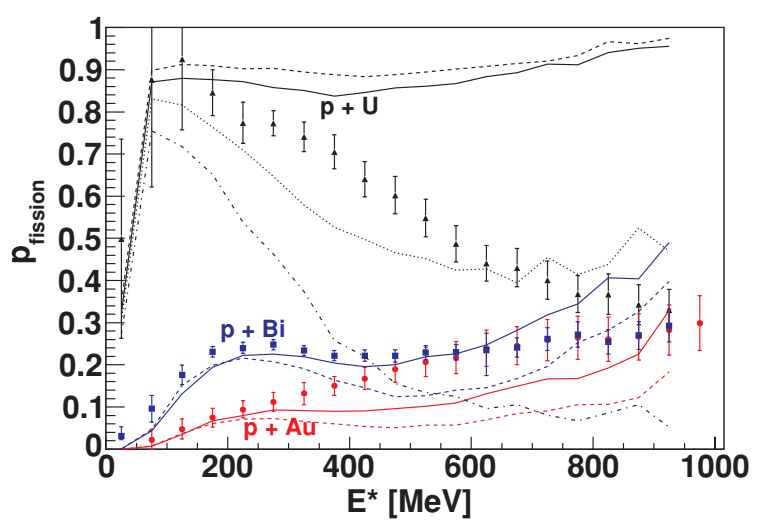

FIG. 19. (Color online) Fission probability as a function of the excitation energy of remnants in $2.5-\mathrm{GeV} p+\mathrm{Au}, \mathrm{Bi}$, and $\mathrm{U}$ reactions. Solid lines: Bohr-Wheeler width, $a_{\mathrm{f}} / a_{\mathrm{n}}=1.036$. Dashed lines: Lestone, $a_{\mathrm{f}} / a_{\mathrm{n}}=1.057, t_{\text {delay }}=1 \mathrm{zs}$. Dotted line: BohrWheeler width, $a_{\mathrm{f}} / a_{\mathrm{n}}=1.02$. Dash-dotted line: Bohr-Wheeler width, $a_{\mathrm{f}} / a_{\mathrm{n}}=1.00$.
An energy-dependent $a_{\mathrm{f}} / a_{\mathrm{n}}$ ratio can naturally appear as a consequence of, among other things, the fade-out of longrange correlations. To obtain a better reproduction of the the Tishchenko data, we have considered a simple refinement of the formula for the level-density parameter at saddle point, Eq. (2), as follows:

$$
\tilde{a}_{\mathrm{f}}(U)=\frac{A}{k_{\infty}-r\left(k_{\infty}-k_{0}\right) \exp \left(-f_{\left.\frac{\kappa}{k_{\infty}-k_{0}} \frac{U}{A}\right)}\right.} .
$$

The $r$ variable, which replaces the $a_{\mathrm{f}} / a_{\mathrm{n}}$ ratio, is a free parameter that describes the difference in the effect of longrange correlations for the saddle point. In the limit of zero excitation energy, Eq. (4) leads to

$$
\frac{a_{\mathrm{f}}}{a_{\mathrm{n}}}=\frac{k_{0}}{k_{\infty}-r\left(k_{\infty}-k_{0}\right)},
$$

while for $U \rightarrow \infty, a_{\mathrm{f}}=a_{\mathrm{n}}=A / k_{\infty}$. The value of $r$ thus determines the $a_{\mathrm{f}} / a_{\mathrm{n}}$ ratio at low energies. We expect on physical grounds that $r$ should be slightly larger than 1, to reflect the increase in surface area and the enhanced collective enhancement of the saddle-point configuration. This would also lead to $a_{\mathrm{f}} / a_{\mathrm{n}}>1$ at small $U$. The parameter $f$, in contrast, expresses the different fade-out rate of long-range correlations at the saddle point compared to the ground state. This quantity is essentially unconstrained by experimental data. However, we observe, from Sec. III B, that the approximation of an energy-independent $a_{\mathrm{f}} / a_{\mathrm{n}}$ ratio is a good one at low excitation energies, as we can successfully reproduce fission cross sections in fusion and spallation. We impose this condition by requiring that

$$
\left.\frac{\partial\left(a_{\mathrm{f}} / a_{\mathrm{n}}\right)}{\partial U}\right|_{U=0}=0
$$

This introduces a correlation between the $f$ and $r$ parameters:

$$
f=\frac{k_{\infty}-r\left(k_{\infty}-k_{0}\right)}{r k_{0}} .
$$

There is no a priori reason to expect that the fade-out rate at the saddle point (described by $f$ ) should be correlated with the $a_{\mathrm{f}} / a_{\mathrm{n}}$ ratio at low energies. We make this assumption on a phenomenological basis. Note that Eq. (5) implies that $f<1$ for $r>1$, that is, that long-range correlations should fade out more slowly at the saddle point than in the ground state. One should also note that $\kappa$ has a very strong mass dependence [5], and therefore the modification of $a_{\mathrm{f}} / a_{\mathrm{n}}$ with excitation energy is much stronger for the $p+\mathrm{U}$ reaction compared to the lighter systems. One can indeed see from Fig. 19 that this is the system that requires the greatest modification from our previous solution.

We can, finally, determine the value of the $r$ parameter by requiring, for example, that the fission cross section for $1-\mathrm{GeV}$ $p+{ }^{208} \mathrm{~Pb}$ be correctly reproduced. For a Bohr-Wheeler width without fission delay, this condition yields $r=1.0747$, which corresponds to $a_{\mathrm{f}} / a_{\mathrm{n}}=1.051$ for $U=0$. For a Lestone width with a 1-zs fission delay, we get $r=1.1$ and $a_{\mathrm{f}} / a_{\mathrm{n}}=1.069$ at $U=0$. Fusion-fission and spallation-fission are not severely affected by this modification, as shown by the dotted and dashed-dotted curves in Figs. 4-11. The resulting fission 


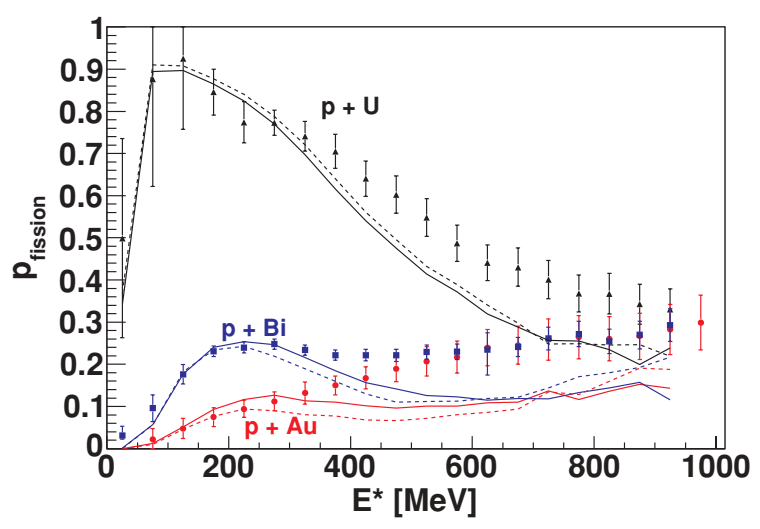

FIG. 20. (Color online) Same as Fig. 19, for a Bohr-Wheeler width (solid lines) or a Lestone width with a 1-zs fission delay (dashed lines) and an energy-dependent effective $a_{\mathrm{f}} / a_{\mathrm{n}}$ ratio, Eq. (4).

probability curves for the $2.5-\mathrm{GeV}$ reactions are shown in Fig. 20. We have good quantitative agreement up to $\sim 400 \mathrm{MeV}$, and we can qualitatively reproduce the decrease in fission probability with excitation energy for the uranium target. This proves that the fission probability at very high excitation energies is indeed sensitive to the fade-out of collective effects at the saddle point.

We have thus shown that one can obtain a similar quality of agreement with or without a fission delay, provided that one increases the value of $r$. Therefore, while we agree with Tishchenko et al. [13] that no transients are needed to explain their data, one cannot rule out the presence of fission transients as well. However, the preceding calculations and conclusions should be taken with caution. Level densities and fission probabilities are dramatically sensitive to all their ingredients at such high excitation energies. We have indeed observed that the shape of the curve can also be modified, for example, by introducing surface terms $\left(\propto A^{2 / 3}\right)$ in the level-density parameter formula or by considering different functional forms for the fade-out of long-range correlations. Even the competition with evaporation cannot be neglected. Finally, very high excitation energies will eventually give rise to other phenomena, such as nuclear expansion and multifragmentation, which are not accounted for in our framework. With all these considerations in mind, we conclude that pursuing perfect agreement between calculations and measurements of fission probabilities for $E^{*} \gtrsim 500 \mathrm{MeV}$ is useless for our understanding of the physics of de-excitation.

\section{CONCLUSIONS}

We have described the first coupling of the INCL model with the GEMINI++ compound-nucleus de-excitation model. The fission probability was calculated using Sierk's finiterange liquid-drop fission barriers [25] and the excitationenergy-dependent level-density parameters of Ref. [5] adjusted to reproduce experimental kinetic-energy spectra of light particles. The latter were very important for obtaining the correct excitation-energy dependence for heavy systems. It was demonstrated (Sec. III) that it is possible to describe fission cross sections from spallation and heavy-ion fusion reactions for $160<A<230$ within the same framework.
Spallation and fusion reactions populate different regions of the compound-nucleus parameter space, and thus they probe different, but overlapping areas of the model-parameter space. Thus, simultaneous fitting of the statistical-decay model parameters to spallation and fusion actually allows one to lift some of the degeneracy. However, even with the large range of spin and excitation energies studied, no unique parameter set could be obtained and there remained some ambiguities in the choice of parameters. In particular, the effect of an increasing fission delay associated with fission transients could be offset by an increase in the $a_{\mathrm{f}} / a_{\mathrm{n}}$ parameter, the ratio of level-density parameters in the saddle-point and ground-state configurations. In addition, modifications to the height of Sierk's fission barrier could be offset by scaling of the fission decay width, which could be associated with the Kramers scaling of the Bohr-Wheeler decay width owing to friction. Despite these ambiguities, we present two sets of statistical model parameters suitable for predictions of fission probabilities for spins up to $60 \hbar$ and excitation energies up to $\sim 300 \mathrm{MeV}$.

From study of the width of the fragment-fragment mass distributions in both fusion and spallation reactions, we were able to differentiate the systematics compiled by Rusanov et al. based on thermal distributions at either the saddle or the scission point (Sec. III C). Only the saddle-point systematics provided a good reproduction of the experimental data in both types of reactions, thus suggesting that the fission mass division is determined close to the saddle point. The asymmetry dependence of the saddle-point conditional barriers in the Rusanov systematics is stronger than that of Sierk's prediction, which produces very wide fission-fragment mass distributions when incorporated in the Moretto formalism. Further indications could, in principle, be extracted from the study of other observables, such as pre- and postscission neutron multiplicities. However, this would happen at the expense of introducing new parameters and ingredients for the description of the saddle-to-scission dynamics, which would be difficult to constrain owing to the lack of relevant data for spallation reactions.

We have proven (Sec. IV) that we can qualitatively describe fission probabilities at excitation energies higher than $300 \mathrm{MeV}$ by accommodating different fade-out rates for the ground-state and the saddle-point configurations. However, we cannot exclude that other solutions are possible, given the uncontrollable sensitivity of the predictions of the model to a large number of its ingredients. Thus, we conclude that the theoretical uncertainties in fission probabilities at very high excitation energies are too large to permit drawing strong conclusions about the physics of highly excited nuclei.

\section{ACKNOWLEDGMENTS}

The authors wish to thank S. Leray for commenting on the manuscript, K.-H. Schmidt for useful discussions, and J. Benlliure for kindly providing experimental data for the $p+{ }^{181} \mathrm{Ta}$ system. This work was supported by the US Department of Energy, Division of Nuclear Physics, under Grant No. DE-FG02-87ER-40316 and by the European Union IP EUROTRANS project (EU Contract No. FI6W-CT-2004516520). 
[1] N. Bohr and J. A. Wheeler, Phys. Rev. 56, 426 (1939).

[2] V. F. Weisskopf and D. H. Ewing, Phys. Rev. 57, 472 (1940).

[3] L. G. Moretto, Nucl. Phys. A 247, 211 (1975).

[4] W. J. Światecki, Aust. J. Phys. 36, 641 (1983).

[5] R. J. Charity, Phys. Rev. C 82, 014610 (2010).

[6] P. Grangé, J.-Q. Li, and H. A. Weidenmüller, Phys. Rev. C 27, 2063 (1983).

[7] D. Hilscher and H. Rossner, Ann. Phys. (Paris) 17, 471 (1992).

[8] R. J. Charity, arXiv:nucl-th/0406040v1 (2004).

[9] P. Fröbrich, I. I. Gontchar, and N. D. Mavlitov, Nucl. Phys. A 556, 281 (1993).

[10] B. B. Back, D. J. Blumenthal, C. N. Davids, D. J. Henderson, R. Hermann, D. J. Hofman, C. L. Jiang, H. T. Penttilä, and A. H. Wuosmaa, Phys. Rev. C 60, 044602 (1999).

[11] L. G. Moretto, K. X. Jing, R. Gatti, G. J. Wozniak, and R. P. Schmitt, Phys. Rev. Lett. 75, 4186 (1995).

[12] J. P. Lestone and S. G. McCalla, Phys. Rev. C 79, 044611 (2009).

[13] V. Tishchenko, C.-M. Herbach, D. Hilscher, U. Jahnke, J. Galin, F. Goldenbaum, A. Letourneau, and W.-U. Schröder, Phys. Rev. Lett. 95, 162701 (2005).

[14] R. J. Charity, in Joint ICTP IAEA Advanced Workshop on Model Codes for Spallation Reactions, Report INDC(NDC)0530 (IAEA, Trieste, Italy, 2008), p. 139.

[15] A. Boudard, J. Cugnon, S. Leray, and C. Volant, Phys. Rev. C 66, 044615 (2002).

[16] R. J. Charity et al., Nucl. Phys. A 483, 371 (1988).

[17] W. Hauser and H. Feshbach, Phys. Rev. 87, 366 (1952).

[18] A. J. Sierk, Phys. Rev. Lett. 55, 582 (1985).

[19] N. Carjan and J. M. Alexander, Phys. Rev. C 38, 1692 (1988).

[20] H. Y. Han, K. X. Jing, E. Plagnol, D. R. Bowman, R. J. Charity, L. Vinet, G. J. Wozniak, and L. G. Moretto, Nucl. Phys. A 492, 138 (1989).

[21] A. Y. Rusanov, M. G. Itkis, and V. N. Okolovich, Phys. At. Nucl. 60, 683 (1997).

[22] A. V. Ignatyuk, G. N. Smirenkin, and A. S. Tishin, Sov. J. Nucl. Phys. 21, 255 (1975).

[23] J. Cugnon, A. Boudard, S. Leray, and D. Mancusi (in preparation).

[24] J. Cugnon, A. Boudard, S. Leray, and D. Mancusi, in International Conference on Nuclear Data for Science and Technology (Korean Nuclear Society and Korean Atomic Energy Research Institute, Jeju Island, 2010).

[25] A. J. Sierk, Phys. Rev. C 33, 2039 (1986).

[26] R. J. Charity et al., Nucl. Phys. A 457, 441 (1986).

[27] K. T. Lesko, W. Henning, K. E. Rehm, G. Rosner, J. P. Schiffer, G. S. F. Stephans, B. Zeidman, and W. S. Freeman, Phys. Rev. C 34, 2155 (1986).

[28] D. Ackermann et al., J. Phys. G 23, 1167 (1997).

[29] R. V. F. Janssens, R. Holzmann, W. Henning, T. L. Khoo, K. T. Lesko, G. S. F. Stephans, D. C. Radford, A. M. V. D. Berg, W. Kühn, and R. M. Ronningen, Phys. Lett. B 181, 16 (1986).

[30] F. L. H. Wolfs, R. V. F. Janssens, R. Holzmann, T. L. Khoo, W. C. Ma, and S. J. Sanders, Phys. Rev. C 39, 865 (1989).

[31] R. J. Charity et al., Phys. Rev. C 67, 044611 (2003).

[32] R. J. Charity, J. F. Dempsey, R. Popelka, and L. G. Sobotka, unpublished data (2000).

[33] B. J. Fineman, K.-T. Brinkmann, A. L. Caraley, N. Gan, R. L. McGrath, and J. Velkovska, Phys. Rev. C 50, 1991 (1994).
[34] D. J. Hinde, J. R. Leigh, J. O. Newton, W. Galster, and S. Sie, Nucl. Phys. A 385, 109 (1982).

[35] A. L. Caraley, B. P. Henry, J. P. Lestone, and R. Vandenbosch, Phys. Rev. C 62, 054612 (2000).

[36] J. G. Keller, B. B. Back, B. G. Glagola, D. Henderson, S. B. Kaufman, S. J. Sanders, R. H. Siemssen, F. Videbaek, B. D. Wilkins, and A. Worsham, Phys. Rev. C 36, 1364 (1987).

[37] A. C. Berriman, D. J. Hinde, M. Dasgupt, C. R. Morton, R. D. Butt, and J. O. Newton, Nature (London) 413, 144 (2001).

[38] K.-T. Brinkmann, A. L. Caraley, B. J. Fineman, N. Gan, J. Velkovska, and R. L. McGrath, Phys. Rev. C 50, 309 (1994).

[39] F. Videbæk, R. B. Goldstein, L. Grodzins, S. G. Steadman, T. A. Belote, and J. D. Garrett, Phys. Rev. C 15, 954 (1977).

[40] B. B. Back, R. R. Betts, J. E. Gindler, B. D. Wilkins, S. Saini, M. B. Tsang, C. K. Gelbke, W. G. Lynch, M. A. McMahan, and P. A. Baisden, Phys. Rev. C 32, 195 (1985).

[41] M. Blann et al., Phys. Rev. C 26, 1471 (1982).

[42] J. Benlliure et al., Nucl. Phys. A 683, 513 (2001).

[43] T. Enqvist et al., Nucl. Phys. A 686, 481 (2001).

[44] M. Bernas et al., Nucl. Phys. A 725, 213 (2003).

[45] B. Fernández-Domínguez et al., Nucl. Phys. A 747, 227 (2005).

[46] K.-H. Schmidt, in Proceedings of the 2007 International Conference on Nuclear Data for Science and Technology (ND2007) (EDP Sciences, Nice, France, 2008).

[47] J. Tõke and W. Światecki, Nucl. Phys. A 372, 141 (1981).

[48] H. A. Kramers, Physica 7, 284 (1940).

[49] J. P. Lestone, Phys. Rev. C 59, 1540 (1999).

[50] J. Benlliure et al., in International Conference on Nuclear Data for Science and Technology (Korean Nuclear Society and Korean Atomic Energy Research Institute, Jeju Island, 2010).

[51] L. Audouin et al., Nucl. Phys. A 768, 1 (2006).

[52] J. Taïeb et al., Nucl. Phys. A 724, 413 (2003).

[53] M. V. Ricciardi et al., Phys. Rev. C 73, 014607 (2006).

[54] R. Rafiei, R. G. Thomas, D. J. Hinde, M. Dasgupta, C. R. Morton, L. R. Gasques, M. L. Brown, and M. D. Rodriguez, Phys. Rev. C 77, 024606 (2008).

[55] B. D. Wilkins, E. P. Steinberg, and R. R. Chasman, Phys. Rev. C 14, 1832 (1976).

[56] A. V. Karpov, P. N. Nadtochy, D. V. Vanin, and G. D. Adeev, Phys. Rev. C 63, 054610 (2001).

[57] E. Ryabov, A. Karpov, and G. Adeev, Nucl. Phys. A 765, 39 (2006).

[58] F. Plasil, D. S. Burnett, H. C. Britt, and S. G. Thompson, Phys. Rev. 142, 696 (1966).

[59] T. Sikkeland, Phys. Rev. 135, B669 (1964).

[60] R. J. Charity et al., Nucl. Phys. A 511, 59 (1990).

[61] L. G. Sobotka et al., Nucl. Phys. A 471, 131 (1987).

[62] V. E. Viola, K. Kwiatkowski, and M. Walker, Phys. Rev. C 31, 1550 (1985).

[63] K. Thomas, R. Davies, and A. J. Sierk, Phys. Rev. C 31, 915 (1985).

[64] K. X. Jing, L. W. Phair, L. G. Moretto, T. Rubehn, L. Beaulieu, T. S. Fan, and G. J. Wozniak, Phys. Lett. B 518, 221 (2001).

[65] V. Tishchenko, C.-M. Herbach, D. Hilscher, U. Jahnke, J. Galin, F. Goldenbaum, A. Letourneau, and W.-U. Schröder, Phys. Rev. Lett. 95, 162701 (2005). 